\title{
Improved approximation rates for a parabolic control problem with an objective promoting directional sparsity
}

\author{
Eduardo Casas · Mariano Mateos · Arnd \\ Rösch
}

Received: date / Accepted: date

\begin{abstract}
We discretize a directionally sparse parabolic control problem governed by a linear equation by means of control approximations that are piecewise constant in time and continuous piecewise linear in space. By discretizing the objective functional with the help of appropriate numerical quadrature formulas, we are able to show that the discrete optimal solution exhibits a directional sparse pattern alike the one enjoyed by the continuous solution. Error estimates are obtained and a comparison with the cases of having piecewise approximations of the control or a semilinear state equation are discussed. Numerical experiments that illustrate the theoretical results are included.
\end{abstract}

Keywords Optimal control, parabolic equations, directionally sparse solutions, finite element approximation, numerical quadrature, error estimates.

Mathematics Subject Classification (2000) Primary: 49K20, 35K58, 65M15; Secondary: 49J52.

The first two authors were partially supported by the Spanish Ministerio de Economía y Competitividad under projects MTM2014-57531-P and MTM2017-83185-P.

Eduardo Casas

Departamento de Matemática Aplicada y Ciencias de la Computación,

E.T.S.I. Industriales y de Telecomunicación, Universidad de Cantabria, 39005 Santander, Spain

E-mail: eduardo.casas@unican.es

Mariano Mateos

Departamento de Matemáticas

Campus de Gijón, Universidad de Oviedo

33203, Gijón, Spain

E-mail: mmateos@uniovi.es

Arnd Rösch

Fakultät für Mathematik

Universtät Duisburg-Essen

D-45127 Essen, Germany

E-mail: arnd.roesch@uni-due.de 


\section{Introduction}

Sparsity promoting techniques in optimal control are of great practical importance. A sparse control is usually easier and cheaper to implement physically than another one that does not have this property. Moreover, a small support for the control is essential in many practical implementations. An important issue is to determine the small region from where the action of the control is the most efficient. Consequently, a great effort has been made during the last years to develop mathematical techniques that lead to problems that have sparse solutions and to solve them.

In [26] it is proved that a term involving the $L^{1}$-norm of the control in the objective functional leads to a solution with sparse structure. The problem treated in that work is a stationary problem governed by a linear elliptic equation. In $[6$, 7] and [29] the finite element discretization of this kind of problems is studied. An alternative to the $L^{1}$ approach is to use measures as controls. See $[4,13,14,23]$.

Sparse time-dependent problems have been investigated in $[1,5,8-10,12,15$, 16]. In this type of problems, there are different possible formulations leading to optimal controls that have different sparse structures; see [8]. Here, we are interested in controls that are sparse in space, not necessarily in time. Moreover, it is desirable that the sparsity pattern does not change in time. Such kind of sparsity is called directional sparsity. The term was coined in the paper [15], where an optimal control problem governed by a linear parabolic equation is studied; to obtain directional sparsity, the authors introduce a term involving the norm in $L^{1}\left(\Omega ; L^{2}(0, T)\right)$. They describe the sparsity properties of the optimal control as well as a semi-smooth Newton method to solve the problem.

In this work, we continue our study, started in [10], about finite element approximations of problems where the optimal solutions are directionally sparse.

Both in the paper at hand and in [10] the state equation is a parabolic partial differential equation and the functional involves a tracking-type term for the state, a Tikhonov regularization term for the control and a non differentiable term that promotes spatial sparsity. Also in both works, the state and the adjoint state equations are discretized using a discontinuous in time and continuous in space Galerkin scheme, namely dG0-cG1, and the controls are discretized in time using piecewise constant functions.

While in [10] the controls are discretized in space using piecewise constant functions, in this work we discretize the controls using functions that are piecewise constant in time and continuous piecewise linear in space. For easier comparison of the results, we will refer to the space of approximation functions used in [10] as $U_{\sigma}$ and to the space of approximation functions used in this work as $V_{\sigma}$.

The theoretical analysis becomes more involved for functions in $V_{\sigma}$. One of the main consequences of using elements in this space is that, with the usual discretization of the functional, the discrete solution may lose its directionally sparsity properties.

To overcome this difficulty, we approximate the integrals of the cost functional involving the control by a numerical quadrature formula (the analogous in $2 \mathrm{D}$ or $3 \mathrm{D}$ to the well known composite trapezoid rule). Proceeding in this way, we recover the same sparsity pattern exhibited by the continuous solution of the control problem. This sparse structure was also proved in [10] for approximations of the controls by piecewise constant functions in time and space. 
This numerical integration leads to new difficulties in the derivation of error estimates. Finally, we are able to obtain an order of convergence for the $L^{2}(Q)$ error in the control variable of order $O(\tau+h)$ in the case of having a parabolic linear state equation. Numerical experiments in Section 8 confirm this obtained order of convergence. This order seems optimal for our approach, regarding the $H^{1}(Q)$-regularity of the optimal control -see [10, Theorem 3.3]-, which limits the order of convergence we can achieve. This is not a special situation in sparse control; see e.g. [18, Theorem 6.1] or [11, Theorem 2.14], where it is emphasized how the approximation error by discrete functions affects the error estimates of the solutions of different optimal control problems. In [19] a problem similar to ours but without sparsity promoting terms is studied and, as in our case, the optimal control exhibits $H^{1}(Q)$-regularity. If no extra assumptions are done, the order of convergence obtained is also $O(\tau+h)$; see [19, Corollary 5.3].

In [10] an order of $O(\sqrt{\tau}+h)$ is obtained. In that paper, the equation is semilinear. With the technique of proof that we show in this work, it can be proven that the order of convergence for approximations in $U_{\sigma}$ is also $O(\tau+h)$ if the state equation is linear. On the other hand, once we have shown how to deal with elements of $V_{\sigma}$, the proofs of [10] can be adapted to obtain an order of convergence of $O(\sqrt{\tau}+h)$ when the state equation is semilinear. We comment on this in Section 7 .

A close inspection to the proofs of [10], in comparison with our Theorem 6.7 and the corresponding result for the semilinear elliptic case [6] gives some insight on the reason we obtain an order of convergence of just $\sqrt{\tau}$ in time for the case of having a semilinear parabolic equation. In the elliptic case, the second order sufficient optimality conditions do not involve the non-differentiable terms of the functional, and in our Theorem 6.7 we use the linearity of the equation to get rid of this terms in the obtention of error estimates. Nevertheless, second order sufficient optimality conditions for the parabolic case involve the non-differentiable term of the functional, see [10, Theorem 4.2], which is what eventually leads to this order $\sqrt{\tau}$.

In [16] a different approach is followed to obtain directional sparsity. Instead of using functions, the control variable is a measure in $\mathcal{M}\left(\Omega ; L^{2}(0, T)\right)$, whose norm in this space is introduced in the functional. The problem is governed by a linear parabolic equation and the error obtained for the $L^{2}(Q)$ norm of the state is $O(\sqrt{\tau}+h)$.

The plan of the paper is as follows. We introduce the problem in Section 2 and recall some results about the continuous problem in Section 3. The problem is discretized in Section 4. Our main results are in Section 5, where we show the sparsity pattern of the discrete solution, and in Section 6, where we prove the convergence of the discretization and provide error estimates. In Section 7 we comment how to adapt the results of [10] to obtain an error estimate of order $O(\sqrt{\tau}+h)$ in the case of having a semilinear equation and how our methods of proof are also valid to show order of convergence $O(\tau+h)$ for piecewise constant approximations of the control if the equation is linear. Finally, we perform numerical experiments in Section 8 . 


\section{Statement of the problem}

Throughout this paper, $\Omega$ denotes an open, bounded subset of $\mathbb{R}^{n}, 1 \leq n \leq 3$, with boundary $\Gamma$, and $0<T<+\infty$ is fixed. We set $Q=\Omega \times(0, T)$ and $\Sigma=\Gamma \times(0, T)$. The control problem is defined in the way

$$
\text { (P) } \min _{u \in L^{2}(Q)} J(u),
$$

where $J(u)=F(u)+\mu j(u)$ with $\mu>0$,

$$
F(u)=\frac{1}{2} \int_{Q}\left(y_{u}-y_{d}\right)^{2} d x d t+\frac{\nu}{2} \int_{Q} u^{2} d x d t,
$$

$\nu>0$, and

$$
j(u)=\|u\|_{L^{1}\left(\Omega ; L^{2}(0, T)\right)}=\int_{\Omega}\|u(t)\|_{L^{2}(0, T)} d t=\int_{\Omega}\left(\int_{0}^{T} u^{2}(x, t) d t\right)^{1 / 2} d x .
$$

For every $u \in L^{2}(Q)$, we denote $y_{u}$ the solution of

$$
\left\{\begin{array}{l}
\partial_{t} y+A y=f(x, t)+u \text { in } Q \\
y=0 \text { on } \Sigma \\
y(0)=y_{0} \text { in } \Omega .
\end{array}\right.
$$

Here, $A$ is the linear elliptic operator

$$
A y=-\sum_{i, j=1}^{n} \partial_{x_{j}}\left[a_{i j}(x) \partial_{x_{i}} y\right]+c(x, t) y .
$$

We make the following assumptions.

Assumption 1. - The boundary $\Gamma$ is of class $C^{1,1}$ or $\Omega$ is convex. The coefficients $a_{i j} \in C^{0,1}(\bar{\Omega})$ and

$$
\exists \Lambda>0 \text { such that } \sum_{i, j=1}^{n} a_{i j}(x) \xi_{i} \xi_{j} \geq \Lambda|\xi|^{2} \forall x \in \Omega \text { and } \forall \xi \in \mathbb{R}^{n} \text {. }
$$

Assumption 2. - The initial datum $y_{0}$ belongs to $H_{0}^{1}(\Omega), f \in L^{2}(Q)$ and $c \in L^{\infty}(Q)$. We also assume that $y_{d} \in L^{\hat{p}}\left(0, T ; L^{\hat{q}}(\Omega)\right)$ with $\hat{p}, \hat{q} \in[2, \infty]$ and $\frac{1}{\hat{p}}+\frac{n}{2 \hat{q}}<1$.

\section{Some results about the continuous problem.}

Let us recall some results concerning problem $(\mathrm{P})$. Since the equation is linear, the study of the differentiable part of the functional is classical. See e.g. [17] or [28]. Under the Assumptions $1-2$, for every $u \in L^{2}(Q)$ there exists a unique $y_{u} \in$ $Y=H^{2,1}(Q)=L^{2}\left(0, T ; H^{2}(\Omega)\right) \cap H^{1}\left(0, T ; L^{2}(\Omega)\right)$ solution of $(1)$ and the controlto-state mapping is $C^{\infty}$. Also, $F: L^{2}(Q) \longrightarrow \mathbb{R}$ is of class $C^{\infty}$ and for all $u, v$ of $L^{2}(Q)$ we have

$$
F^{\prime}(u) v=\int_{Q}\left(\varphi_{u}+\nu u\right) v d x d t
$$


where $\varphi_{u} \in L^{\infty}(Q) \cap H^{2,1}(Q)$ is the solution of

$$
\left\{\begin{aligned}
-\frac{\partial \varphi}{\partial t}+A^{*} \varphi & =y_{u}-y_{d} & & \text { in } Q \\
\varphi & =0 & & \text { on } \Sigma \\
\varphi(T) & =0 & & \text { in } \Omega
\end{aligned}\right.
$$

$A^{*}$ being the adjoint operator of $A$. Next we state the properties of the nondifferentiable part of the functional. The proof of the following result can be found in [8].

Proposition 3.1 Given $u \in L^{1}\left(\Omega ; L^{2}(0, T)\right)$ the following statements hold. 1. $\lambda \in \partial j(u)$ is equivalent to $\lambda \in L^{\infty}\left(\Omega ; L^{2}(0, T)\right)$ and

$$
\left\{\begin{array}{l}
\|\lambda(x)\|_{L^{2}(0, T)} \leq 1 \quad \text { for a. } a . x \in \Omega_{u}^{0}, \\
\lambda(x, t)=\frac{u(x, t)}{\|u(x)\|_{L^{2}(0, T)}} \text { for a. } a . x \in \Omega_{u} \text { and } t \in(0, T),
\end{array}\right.
$$

where

$$
\Omega_{u}=\left\{x \in \Omega:\|u(x)\|_{L^{2}(0, T)} \neq 0\right\} \quad \text { and } \quad \Omega_{u}^{0}=\Omega \backslash \Omega_{u} .
$$

2. For every $v \in L^{1}\left(\Omega ; L^{2}(0, T)\right)$

$$
j^{\prime}(u ; v)=\int_{\Omega_{u}^{0}}\|v(x)\|_{L^{2}(0, T)} d x+\int_{\Omega_{u}} \frac{1}{\|u(x)\|_{L^{2}(0, T)}} \int_{0}^{T} u v d t d x .
$$

We finish this section stating existence, uniqueness, first order optimality conditions and regularity of the solution. Existence of a solution in $L^{2}(Q)$ can be proved by the usual method of taking a minimizing sequence that is bounded in $L^{2}(Q)$. We omit the details of the proof since it is completely standard. Uniqueness follows from the strict convexity of the functional with respect to $u$. First order optimality conditions and regularity of the solution are proved as in [10, Theorem 3.3].

Theorem 3.2 Problem $(\mathrm{P})$ has a unique solution $\bar{u} \in L^{2}(Q)$. Moreover, this solution belongs to $C(\bar{Q}) \cap H^{1}(Q)$, and there exist unique $\bar{y} \in Y, \bar{\varphi} \in Y \cap C(\bar{Q})$ and $\bar{\lambda} \in$ $\partial j(\bar{u}) \cap C(\bar{Q}) \cap H^{1}(Q)$ such that

$$
\begin{aligned}
& \left\{\begin{array}{cc}
\partial_{t} \bar{y}+A \bar{y}=f(x, t)+\bar{u} & \text { in } Q, \\
\bar{y}=0 & \text { on } \Sigma, \\
\bar{y}(0)=y_{0} & \text { in } \Omega,
\end{array}\right. \\
& \left\{\begin{array}{cc}
-\partial_{t} \bar{\varphi}+A^{*} \bar{\varphi}=\bar{y}-y_{d} & \text { in } Q, \\
\bar{\varphi}=0 & \text { on } \Sigma, \\
\bar{\varphi}(T)=0 & \text { in } \Omega,
\end{array}\right. \\
& \bar{\varphi}+\nu \bar{u}+\mu \bar{\lambda}=0 .
\end{aligned}
$$

Furthermore, the following relations hold for all $(x, t) \in Q$ :

$$
\begin{aligned}
& \|\bar{u}(x)\|_{L^{2}(0, T)}=0 \Leftrightarrow\|\bar{\varphi}(x)\|_{L^{2}(0, T)} \leq \mu, \\
& \bar{\lambda}(x, t)=\left\{\begin{array}{cl}
-\frac{1}{\mu} \bar{\varphi}(x, t) \quad \text { if } x \in \Omega_{\bar{u}}^{0}, \\
\frac{\bar{u}(x, t)}{\|\bar{u}(x)\|_{L^{2}(0, T)}} \text { if } x \in \Omega_{\bar{u} .}
\end{array}\right.
\end{aligned}
$$

Remark 3.3 Notice also that $\bar{u} \equiv 0$ and $\bar{\lambda} \equiv 0$ on $\Sigma$. 


\section{Numerical approximation}

In this section, we will further assume that $\Omega$ is convex. We will discretize both the state and the control using functions that are continuous piecewise linear in space and piecewise constant in time. To this aim, we will consider, cf. $[2$, def. (4.4.13)], a quasi-uniform family of triangulations $\left\{\mathcal{K}_{h}\right\}_{h>0}$ of $\bar{\Omega}$ and a quasiuniform family of partitions of $[0, T], 0=t_{0}<t_{1}<\cdots<t_{N_{\tau}}=T$. We will denote $\Omega_{h}=$ int $\cup_{K \in \mathcal{K}_{h}} K, N_{h}$ and $N_{I, h}$ the number of nodes and interior nodes of $\mathcal{K}_{h}$, $I_{j}=\left(t_{j-1}, t_{j}\right), \tau_{j}=t_{j}-t_{j-1}, \tau=\max \left\{\tau_{j}: 1 \leq j \leq N_{\tau}\right\}$ and $\sigma=(h, \tau)$. We assume that every boundary node of $\Omega_{h}$ is a point of $\Gamma$. Additionally we suppose that the distance $d_{\Gamma}(x) \leq C_{\Gamma} h^{2}$ for every $x \in \Gamma_{h}=\partial \Omega_{h}$, which is always satisfied if $n=2$ and $\Gamma$ is of class $C^{2}$; see, for instance, [24, Section 5.2]. Under this assumption we have that

$$
\left|\Omega \backslash \Omega_{h}\right| \leq C h^{2},
$$

where $|\cdot|$ denotes the Lebesgue measure. In the sequel we denote $Q_{h}=\Omega_{h} \times(0, T)$.

Now we consider the finite dimensional spaces

$$
Y_{h}=\left\{y_{h} \in C(\bar{\Omega}): y_{h \mid K} \in P_{1}(K) \forall K \in \mathcal{K}_{h}, y_{h} \equiv 0 \text { in } \bar{\Omega} \backslash \Omega_{h}\right\},
$$

where $P_{1}(K)$ is the space of polynomials of degree less or equal than 1 on the element $K$, and

$$
\mathcal{Y}_{\sigma}=\left\{y_{\sigma} \in L^{2}\left(0, T ; Y_{h}\right): y_{\sigma \mid I_{j}} \in Y_{h} \forall j=1, \ldots, N_{\tau}\right\} .
$$

The elements of $\mathcal{Y}_{\sigma}$ can be written as

$$
y_{\sigma}=\sum_{j=1}^{N_{\tau}} y_{h, j} \chi_{j}=\sum_{j=1}^{N_{\tau}} \sum_{i=1}^{N_{I, h}} y_{i, j} e_{i} \chi_{j}
$$

where $\left\{e_{i}\right\}_{i=1}^{N_{I, h}}$ is the nodal basis associated to the interior nodes $\left\{x_{i}\right\}_{i=1}^{N_{I, h}}$ of the triangulation and $\chi_{j}$ denotes the characteristic function of the interval $I_{j}=\left(t_{j-1}, t_{j}\right)$. For every $u \in L^{2}\left(Q_{h}\right)$, we define its associated discrete state as the unique element $y_{\sigma} \in \mathcal{Y}_{\sigma}$ such that

$$
\begin{aligned}
& \int_{\Omega_{h}}\left(y_{h, j}-y_{h, j-1}\right) z_{h} d x+\tau_{j} a_{h}\left(y_{h, j}, z_{h}\right)+\int_{I_{j}} \int_{\Omega_{h}} c(x, t) y_{h, j} z_{h} d x d t \\
& =\int_{I_{j}} \int_{\Omega_{h}}(f(x, t)+u) z_{h} d x d t \quad \forall z_{h} \in Y_{h} \text { and all } j=1, \ldots, N_{\tau}, \\
& \int_{\Omega_{h}} y_{h, 0} z_{h} d x=\int_{\Omega_{h}} y_{0} z_{h} d x \forall z_{h} \in Y_{h} .
\end{aligned}
$$

where

$$
a_{h}(y, z)=\int_{\Omega_{h}} \sum_{i, j=1}^{n} a_{i, j} \partial_{x_{i}} y \partial_{x_{j}} z d x \quad \forall y, z \in H^{1}\left(\Omega_{h}\right) .
$$

This corresponds to an implicit Euler scheme. It is proved in $[18,20]$ that there exist $h_{0}>0$ and $\tau_{0}>0$ such that

$$
\left\|y_{\sigma}(u)-y_{u}\right\|_{L^{2}\left(Q_{h}\right)} \leq C\left(\tau+h^{2}\right)\|y\|_{H^{2,1}(Q)} \forall h<h_{0}, \tau<\tau_{0} .
$$


To discretize the controls, we will also use continuous piecewise linear in space and piecewise constant in time functions.

$$
\begin{gathered}
V_{h}=\left\{u_{h} \in C\left(\bar{\Omega}_{h}\right): z_{h \mid K} \in P_{1}(K) \forall K \in \mathcal{K}_{h}\right\}, \\
\mathcal{V}_{\sigma}=\left\{u_{\sigma} \in L^{2}\left(0, T ; V_{h}\right): u_{\sigma \mid I_{j}} \in V_{h} \forall j=1, \ldots, N_{\tau}\right\} .
\end{gathered}
$$

The elements of $\mathcal{V}_{\sigma}$ can be written as

$$
u_{\sigma}=\sum_{j=1}^{N_{\tau}} u_{h, j} \chi_{j}=\sum_{j=1}^{N_{\tau}} \sum_{i=1}^{N_{h}} u_{i, j} e_{i} \chi_{j}=\sum_{i=1}^{N_{h}} u_{\tau, i} e_{i}
$$

where $u_{h, j} \in V_{h}$ for $j=1, \ldots, N_{\tau}, u_{i, j} \in \mathbb{R}$ for $i=1, \ldots, N_{h}$ and $j=1, \ldots, N_{\tau}$. Now $\left\{e_{i}\right\}_{i=1}^{N_{h}}$ is the nodal basis associated to all nodes $\left\{x_{i}\right\}_{i=1}^{N_{h}}$ of the triangulation. Notice that $u_{\tau, i}=\sum_{j=1}^{N_{\tau}} u_{i, j} \chi_{j}$ is a piecewise constant function of time.

To formulate the discrete problem we will use numerical quadrature formulas to approximate the norms appearing in the functional $J$. For every $u_{h}, v_{h} \in V_{h}$, we define

$$
\left(u_{h}, v_{h}\right)_{h}=\sum_{i=1}^{N_{h}} u_{i} v_{i} \int_{\Omega_{h}} e_{i} d x
$$

This leads to the following scalar product in $\mathcal{V}_{\sigma}$ :

$$
\left(u_{\sigma}, v_{\sigma}\right)_{\sigma}=\int_{0}^{T}\left(u_{\sigma}(t), v_{\sigma}(t)\right)_{h} d t=\sum_{j=1}^{N_{\tau}} \sum_{i=1}^{N_{h}} \int_{\Omega_{h}} e_{i} d x \tau_{j} u_{i, j} v_{i, j}
$$

We will denote the related norm as $\left\|u_{\sigma}\right\|_{\sigma}=\left(u_{\sigma}, u_{\sigma}\right)_{\sigma}^{1 / 2}$. We also define

$$
j_{\sigma}\left(u_{\sigma}\right)=\left|u_{\sigma}\right|_{\sigma}=\sum_{i=1}^{N_{h}} \int_{\Omega_{h}} e_{i}(x) d x\left(\sum_{j=1}^{N_{\tau}} \tau_{j} u_{i, j}^{2}\right)^{1 / 2}=\sum_{i=1}^{N_{h}} \int_{\Omega_{h}} e_{i}(x) d x\left\|u_{\tau, i}\right\|_{L^{2}(0, T)} .
$$

The discrete problem reads as

$$
\left(\mathrm{P}_{\sigma}\right) \min _{u_{\sigma} \in U_{\sigma}} J_{\sigma}\left(u_{\sigma}\right)=F_{\sigma}\left(u_{\sigma}\right)+\mu j_{\sigma}\left(u_{\sigma}\right),
$$

where

$$
F_{\sigma}\left(u_{\sigma}\right)=\frac{1}{2} \int_{Q_{h}}\left|y_{\sigma}\left(u_{\sigma}\right)-y_{d}\right|^{2} d x d t+\frac{\nu}{2}\left\|u_{\sigma}\right\|_{\sigma}^{2} .
$$

This numerical quadrature scheme is related to a mass lumping approximation, whose necessity in obtaining component-wise subdifferential relations for sparsity penalties is already pointed out in the dissertations of Pieper [22, Section 4.5.3] and Trautmann [27, Section 6.5]. 
5 Sparsity properties of the discrete solutions.

In this section we analyze the discrete control problem $\left(\mathrm{P}_{\sigma}\right)$. We prove that the unique discrete optimal solution has a sparse structure similar to the one established for the continuous optimal control. This sparsity structure follows from the optimality system. Firstly, we observe that under the assumptions $1-2, F_{\sigma}: \mathcal{V}_{\sigma} \rightarrow \mathbb{R}$ is of class $C^{\infty}$. Moreover, for every $u_{\sigma}, v_{\sigma} \in \mathcal{V}_{\sigma}$, we have that

$$
F_{\sigma}^{\prime}\left(u_{\sigma}\right) v_{\sigma}=\int_{Q_{h}} \varphi_{\sigma} v_{\sigma} d x d t+\nu \sum_{j=1}^{N_{\tau}} \sum_{i=1}^{N_{h}} u_{i, j} v_{i, j} \tau_{j} \int_{\Omega} e_{i} d x
$$

where $\varphi_{\sigma} \in \mathcal{Y}_{\sigma}$ is the discrete adjoint state associated with $u_{\sigma}$. We recall that the discrete adjoint state $\varphi_{\sigma}(u) \in \mathcal{Y}_{\sigma}$ associated with an element $u \in L^{2}\left(Q_{h}\right)$ is the solution of the equation

$$
\begin{aligned}
& \varphi_{N_{\tau}+1, h}=0 \\
& \int_{\Omega_{h}}\left(\varphi_{h, j}-\varphi_{j+1, h}\right) z_{h} d x+\tau_{j} a_{h}\left(z_{h}, \varphi_{h, j}\right)+\int_{I_{j}} \int_{\Omega_{h}} c(x, t) \varphi_{h, j} z_{h} d x d t \\
& =\int_{I_{j}} \int_{\Omega_{h}}\left(y_{h, j}-y_{d}\right) z_{h} d x d t \quad \forall z_{h} \in Y_{h} \text { for all } j=N_{\tau}, \ldots, 1,
\end{aligned}
$$

where $y_{\sigma}=y_{\sigma}(u)$ is the discrete state corresponding to $u$.

We rewrite now $F_{\sigma}^{\prime}\left(u_{\sigma}\right)$ using Carstensen's quasi-interpolation operator, cf. [3], $\Pi_{h}: L^{1}\left(\Omega_{h}\right) \longrightarrow V_{h}$ defined by

$$
\Pi_{h} z=\sum_{i=1}^{N_{h}} \frac{\int_{\Omega_{h}} z e_{i} d x}{\int_{\Omega_{h}} e_{i} d x} e_{i}
$$

Notice that for every $z \in L^{1}\left(\Omega_{h}\right)$ and all $v_{h} \in V_{h}$

$$
\left(\Pi_{h} z, v_{h}\right)_{h}=\int_{\Omega_{h}} z v_{h} d x
$$

Given $\varphi_{\sigma}\left(u_{\sigma}\right)$, we denote

$$
\phi_{i, j}=\frac{\int_{\Omega_{h}} \varphi_{h, j} e_{i} d x}{\int_{\Omega_{h}} e_{i} d x} .
$$

The derivative of the differentiable part of the approximated functional can be expressed as

$$
F_{\sigma}^{\prime}\left(u_{\sigma}\right) v_{\sigma}=\sum_{j=1}^{N_{\tau}} \sum_{i=1}^{N_{h}}\left(\phi_{i, j}+\nu u_{i, j}\right) v_{i, j} \tau_{j} \int_{\Omega_{h}} e_{i} d x .
$$

Let us study the non-differentiable part of the cost functional. First, we compute the directional derivatives. To this end let us set

$$
I_{\sigma}\left(u_{\sigma}\right)=\left\{i: \sum_{j=1}^{N_{\tau}} \tau_{j} u_{i, j}^{2} \neq 0\right\} \quad \text { and } \quad I_{\sigma}^{0}\left(u_{\sigma}\right)=\left\{1, \ldots, N_{h}\right\} \backslash I_{\sigma} .
$$


The directional derivative of $j_{\sigma}$ at a point $u_{\sigma} \in \mathcal{V}_{\sigma}$ in the direction $v_{\sigma} \in \mathcal{V}_{\sigma}$ can be written as

$$
j_{\sigma}^{\prime}\left(u_{\sigma} ; v_{\sigma}\right)=\sum_{i \in I_{\sigma}^{0}} \int_{\Omega} e_{i} d x\left\|v_{\tau, i}\right\|_{L^{2}(0, T)}+\sum_{i \in I_{\sigma}} \int_{\Omega} e_{i} d x \sum_{j=1}^{N_{\tau}} \tau_{j} \frac{u_{i, j} v_{i, j}}{\left\|u_{i}\right\|_{L^{2}(0, T)}}
$$

Now, we focus on the subdifferentiability of $j_{\sigma}$. We have the following characterization of the elements of the subdifferential of $j_{\sigma}\left(u_{\sigma}\right)$.

Proposition 5.1 Every $\lambda_{\sigma} \in \partial j_{\sigma}\left(u_{\sigma}\right) \subset \mathcal{V}_{\sigma}$, w.r.t. to the scalar product $(\cdot, \cdot)_{\sigma}$ defined in (15), satisfies:

$$
\left\{\begin{array}{l}
\sum_{j=1}^{N_{\tau}} \tau_{j} \lambda_{i, j}^{2} \leq 1 \quad \forall i \in I_{\sigma}^{0}\left(u_{\sigma}\right) \\
\lambda_{i, j}=\frac{u_{i, j}}{\left\|u_{i}\right\|_{L^{2}(0, T)}} \quad \forall i \in I_{\sigma}\left(u_{\sigma}\right) \text { and } 1 \leq j \leq N_{\tau}
\end{array}\right.
$$

Conversely, every $\lambda_{\sigma} \in \mathcal{V}_{\sigma}$ satisfying (19) is a subgradient of $j_{\sigma}\left(u_{\sigma}\right)$ w.r.t. $(\cdot, \cdot)_{\sigma}$.

Proof Suppose $\lambda_{\sigma} \in \partial j_{\sigma}\left(u_{\sigma}\right)$. Then we have by definition of subdifferential

$$
\left(\lambda_{\sigma}, v_{\sigma}-u_{\sigma}\right)_{\sigma}+j_{\sigma}\left(u_{\sigma}\right) \leq j_{\sigma}\left(v_{\sigma}\right) \forall v_{\sigma} \in \mathcal{V}_{\sigma},
$$

which means

$$
\begin{gathered}
\sum_{j=1}^{N_{\tau}} \sum_{k=1}^{N_{h}} \int_{\Omega_{h}} e_{k}(x) d x \tau_{j} \lambda_{k, j}\left(v_{k, j}-u_{k, j}\right)+\sum_{k=1}^{N_{h}} \int_{\Omega_{h}} e_{k}(x) d x\left(\sum_{j=1}^{N_{\tau}} \tau_{j} u_{k, j}^{2}\right)^{1 / 2} \\
\leq \sum_{k=1}^{N_{h}} \int_{\Omega_{h}} e_{k}(x) d x\left(\sum_{j=1}^{N_{\tau}} \tau_{j} v_{k, j}^{2}\right)^{1 / 2} \forall \mathbf{v}=\left(v_{k, j}\right) \in \mathbb{R}^{N_{h} \times N_{\tau}}
\end{gathered}
$$

Fix some $i \in\left\{1, \cdots, N_{h}\right\}$ and take $v_{k, j}=u_{k, j}$ if $k \neq i$. We obtain

$$
\sum_{j=1}^{N_{\tau}} \tau_{j} \lambda_{i, j}\left(v_{i, j}-u_{i, j}\right)+\left(\sum_{j=1}^{N_{\tau}} \tau_{j} u_{i, j}^{2}\right)^{1 / 2} \leq\left(\sum_{j=1}^{N_{\tau}} \tau_{j} v_{i, j}^{2}\right)^{1 / 2} \forall \mathbf{v}_{i, \cdot} \in \mathbb{R}^{N_{\tau}}
$$

with $\mathbf{v}_{i, \cdot}=\left(v_{i, j}\right)_{j=1}^{N_{\tau}}$. Suppose first that $i \in I_{\sigma}^{0}\left(u_{\sigma}\right)$. Then

$$
\sum_{j=1}^{N_{\tau}} \tau_{j} \lambda_{i, j} v_{i, j} \leq\left(\sum_{j=1}^{N_{\tau}} \tau_{j} v_{i, j}^{2}\right)^{1 / 2} \forall \mathbf{v}_{i, \cdot} \in \mathbb{R}^{N_{\tau}}
$$

Taking $v_{i, j}=\lambda_{i, j}$, we get

$$
\sum_{j=1}^{N_{\tau}} \tau_{j} \lambda_{i, j}^{2} \leq\left(\sum_{j=1}^{N_{\tau}} \tau_{j} \lambda_{i, j}^{2}\right)^{1 / 2}
$$

which implies that $\sum_{j=1}^{N_{\tau}} \tau_{j} \lambda_{i, j}^{2} \leq 1$. 
Let us consider now the case of $i \in I_{\sigma}\left(u_{\sigma}\right)$. Noticing that

$$
(\mathbf{a}, \mathbf{b})_{\tau}=\sum_{j=1}^{N_{\tau}} \tau_{j} a_{j} b_{j}
$$

is a scalar product in $\mathbb{R}^{N_{\tau}}$, whose related norm we denote $|\cdot|_{\tau}$, we have that relation (20) is equivalent to $\boldsymbol{\lambda}_{i,}, \in \partial|\cdot|_{\tau}\left(\mathbf{u}_{i, .}\right)$. Since $i \in I_{\sigma}\left(u_{\sigma}\right)$ means that $\left|\mathbf{u}_{i, .}\right|_{\tau} \neq 0$, the subdifferential is a singleton and an elementary computation of the partial derivatives yields the expression claimed for $\lambda_{i, j}$.

Suppose now that $\lambda_{\sigma}$ satisfies (19). If $i \in I_{\sigma}^{0}\left(u_{\sigma}\right)$, then $\sum_{j=1}^{N_{\tau}} \tau_{j} \lambda_{i, j}^{2} \leq 1$, and using the Cauchy-Schwarz inequality for the scalar product defined in (21), we have that

$$
\sum_{j=1}^{N_{\tau}} \tau_{j} \lambda_{i, j} v_{i, j} \leq\left(\sum_{j=1}^{N_{\tau}} \tau_{j} v_{i, j}^{2}\right)^{1 / 2} \forall \mathbf{v}_{i, \cdot} \in \mathbb{R}^{N_{\tau}}
$$

and hence

$$
\begin{aligned}
& \sum_{i \in I_{\sigma}^{0}\left(u_{\sigma}\right)} \int_{\Omega_{h}} e_{i}(x) d x \sum_{j=1}^{N_{\tau}} \tau_{j} \lambda_{i, j} v_{i, j} \\
\leq & \sum_{i \in I_{\sigma}^{0}\left(u_{\sigma}\right)} \int_{\Omega_{h}} e_{i}(x) d x\left(\sum_{j=1}^{N_{\tau}} \tau_{j} v_{i, j}^{2}\right)^{1 / 2} \forall \mathbf{v}_{\cdot, \cdot}, \in \mathbb{R}^{I_{\sigma}^{0}\left(u_{\sigma}\right) \times N_{\tau}} .
\end{aligned}
$$

In the case $i \in I_{\sigma}\left(u_{\sigma}\right)$, we deduce from (19) that

$$
\sum_{j=1}^{N_{\tau}} \tau_{j} \lambda_{i, j} u_{i, j}=\left(\sum_{j=1}^{N_{\tau}} \tau_{j} u_{i, j}^{2}\right)^{1 / 2}
$$

From the Cauchy-Schwarz inequality and (19), we have

$$
\begin{aligned}
\sum_{j=1}^{N_{\tau}} \tau_{j} \lambda_{i, j} v_{i, j} & \leq\left(\sum_{j=1}^{N_{\tau}} \tau_{j} v_{i, j}^{2}\right)^{1 / 2}\left(\sum_{j=1}^{N_{\tau}} \tau_{j} \lambda_{i, j}^{2}\right)^{1 / 2} \\
& =\left(\sum_{j=1}^{N_{\tau}} \tau_{j} v_{i, j}^{2}\right)^{1 / 2}\left(\sum_{j=1}^{N_{\tau}} \tau_{j} \frac{u_{i, j}^{2}}{\sum_{k=1}^{N_{\tau}} \tau_{k} u_{i, k}^{2}}\right)^{1 / 2} \\
& =\left(\sum_{j=1}^{N_{\tau}} \tau_{j} v_{i, j}^{2}\right)^{1 / 2}
\end{aligned}
$$

From this and (23), we obtain that if $i \in I_{\sigma}\left(u_{\sigma}\right)$ then

$$
\sum_{j=1}^{N_{\tau}} \tau_{j} \lambda_{i, j}\left(v_{i, j}-u_{i, j}\right)+\left(\sum_{j=1}^{N_{\tau}} \tau_{j} u_{i, j}^{2}\right)^{1 / 2} \leq\left(\sum_{j=1}^{N_{\tau}} \tau_{j} v_{i, j}^{2}\right)^{1 / 2} \forall \mathbf{v}_{i, \cdot} \in \mathbb{R}^{N_{\tau}}
$$


and hence

$$
\begin{aligned}
& \sum_{i \in I_{\sigma}\left(u_{\sigma}\right)} \int_{\Omega_{h}} e_{i}(x) d x \sum_{j=1}^{N_{\tau}} \tau_{j} \lambda_{i, j}\left(v_{i, j}-u_{i, j}\right) \\
& +\sum_{i \in I_{\sigma}\left(u_{\sigma}\right)} \int_{\Omega_{h}} e_{i}(x) d x\left(\sum_{j=1}^{N_{\tau}} \tau_{j} u_{i, j}^{2}\right)^{1 / 2} \\
& \leq \sum_{i \in I_{\sigma}\left(u_{\sigma}\right)} \int_{\Omega_{h}} e_{i}(x) d x\left(\sum_{j=1}^{N_{\tau}} \tau_{j} v_{i, j}^{2}\right)^{1 / 2} \forall \mathbf{v} \cdot, \in \mathbb{R}^{I_{\sigma}\left(u_{\sigma}\right) \times N_{\tau}} .
\end{aligned}
$$

Adding this inequality and (22), we obtain that $\lambda_{\sigma}$ satisfies the subgradient condition.

In the sequel we denote $J_{\sigma}^{\prime}\left(u_{\sigma} ; v_{\sigma}\right)=F_{\sigma}^{\prime}\left(u_{\sigma}\right) v_{\sigma}+\mu j_{\sigma}^{\prime}\left(u_{\sigma} ; v_{\sigma}\right)$. First order optimality conditions follow in a natural way from the convexity of $j_{\sigma}$, the definition of subdifferential, (16) and (17):

Theorem 5.2 Problem $\left(\mathrm{P}_{\sigma}\right)$ has a unique solution $\bar{u}_{\sigma} \in \mathcal{V}_{\sigma}$ and there exist unique $\bar{y}_{\sigma}=y_{\sigma}\left(\bar{u}_{\sigma}\right), \bar{\varphi}_{\sigma}=\varphi_{\sigma}\left(\bar{u}_{\sigma}\right) \in \mathcal{Y}_{\sigma}$ and $\bar{\lambda}_{\sigma} \in \partial j_{\sigma}\left(\bar{u}_{\sigma}\right)$ such that

$$
\Pi_{h} \bar{\varphi}_{\sigma}+\nu \bar{u}_{\sigma}+\mu \bar{\lambda}_{\sigma}=0 .
$$

Moreover the inequality $J_{\sigma}^{\prime}\left(\bar{u}_{\sigma} ; v_{\sigma}\right) \geq 0$ holds $\forall v_{\sigma} \in \mathcal{V}_{\sigma}$.

Proof Existence of solution follows from the coercivity and continuity of $J_{\sigma}$ and the uniqueness is a consequence of its strict convexity. From the optimality of $\bar{u}_{\sigma}$ and the convexity of $j_{\sigma}\left(u_{\sigma}\right)$, we have that

$$
\begin{aligned}
0 \leq & \lim _{\rho \searrow 0} \frac{J_{\sigma}\left(\bar{u}_{\sigma}+\rho\left(u_{\sigma}-\bar{u}_{\sigma}\right)\right)-J\left(\bar{u}_{\sigma}\right)}{\rho} \\
= & \lim _{\rho \searrow 0} \frac{F_{\sigma}\left(\bar{u}_{\sigma}+\rho\left(u_{\sigma}-\bar{u}_{\sigma}\right)\right)-F_{\sigma}\left(\bar{u}_{\sigma}\right)}{\rho} \\
& +\mu \lim _{\rho \searrow 0} \frac{j_{\sigma}\left(\bar{u}_{\sigma}+\rho\left(u_{\sigma}-\bar{u}_{\sigma}\right)\right)-j_{\sigma}\left(\bar{u}_{\sigma}\right)}{\rho} \\
= & F_{\sigma}^{\prime}\left(\bar{u}_{\sigma}\right)\left(u_{\sigma}-\bar{u}_{\sigma}\right)+\mu j_{\sigma}^{\prime}\left(\bar{u}_{\sigma} ; u_{\sigma}-\bar{u}_{\sigma}\right) \\
\leq & F_{\sigma}^{\prime}\left(\bar{u}_{\sigma}\right)\left(u_{\sigma}-\bar{u}_{\sigma}\right)+\mu j_{\sigma}\left(u_{\sigma}\right)-\mu j_{\sigma}\left(\bar{u}_{\sigma}\right) \quad \forall u_{\sigma} \in \mathcal{V}_{\sigma} .
\end{aligned}
$$

This proves that $J_{\sigma}^{\prime}\left(\bar{u}_{\sigma} ; v_{\sigma}\right) \geq 0 \forall v_{\sigma} \in \mathcal{V}_{\sigma}$. To obtain (24), first we observe that the previous chain of inequalities implies that

$$
-\frac{1}{\mu} F_{\sigma}^{\prime}\left(\bar{u}_{\sigma}\right)\left(u_{\sigma}-\bar{u}_{\sigma}\right)+j_{\sigma}\left(\bar{u}_{\sigma}\right) \leq j_{\sigma}\left(u_{\sigma}\right) \quad \forall u_{\sigma} \in \mathcal{V}_{\sigma}
$$

Taking into account the definition (15) of the scalar product $(\cdot, \cdot)_{\sigma}$, the expression for the derivative (16) and the property (17) of Carstensen's quasi-interpolation operator, we have that for any $u_{\sigma} \in \mathcal{V}_{\sigma}$ we have that

$$
F_{\sigma}^{\prime}\left(\bar{u}_{\sigma}\right) u_{\sigma}=\left(\Pi_{h} \bar{\varphi}_{\sigma}+\nu \bar{u}_{\sigma}, u_{\sigma}\right)_{\sigma} .
$$

Finally, from (25) and the definition of subdifferential we infer that $-\frac{1}{\mu}\left(\Pi_{h} \bar{\varphi}_{\sigma}+\right.$ $\left.\nu \bar{u}_{\sigma}\right) \in \partial j_{\sigma}\left(\bar{u}_{\sigma}\right)$ and, hence, (24) follows. The uniqueness of $\bar{\lambda}_{\sigma}$ follows from (24). 
For a local minimum $\bar{u}_{\sigma} \in \mathcal{V}_{\sigma}$ with related discrete adjoint state $\bar{\varphi}_{\sigma}$, we will denote the components of Cartensen's quasi interpolation of $\bar{\varphi}_{h, j}$ as

$$
\bar{\phi}_{i, j}=\frac{\int_{\Omega_{h}} \bar{\varphi}_{h, j} e_{i} d x}{\int_{\Omega_{h}} e_{i} d x}
$$

i.e. $\Pi_{h} \bar{\varphi}_{\sigma}=\sum_{j=1}^{N_{\tau}} \sum_{i=1}^{N_{h}} \bar{\phi}_{i, j} e_{i} \chi_{j}$. We finish this section by characterizing $\bar{\lambda}_{\sigma}$ and proving the sparsity of $\bar{u}_{\sigma}$.

Theorem 5.3 Let $\bar{u}_{\sigma}$ be the solution of $\left(\mathrm{P}_{\sigma}\right)$. Then, the element $\bar{\lambda}_{\sigma} \in \partial j_{\sigma}\left(\bar{u}_{\sigma}\right)$ satisfying (24) can be written as

$$
\bar{\lambda}_{i, j}=\left\{\begin{array}{cl}
\frac{-\bar{\phi}_{i, j}}{\mu} & \text { if } j \in I_{\sigma}^{0}\left(\bar{u}_{\sigma}\right) \\
\frac{\bar{u}_{i, j}}{\left\|\bar{u}_{i}\right\|_{L^{2}(0, T)}} & \text { if } i \in I_{\sigma}\left(\bar{u}_{\sigma}\right) .
\end{array}\right.
$$

The optimal control satisfies

$$
\bar{u}_{i, j}\left[\nu+\frac{\mu}{\left\|\bar{u}_{i}\right\|_{L^{2}(0, T)}}\right]=-\bar{\phi}_{i, j} \text { if } i \in I_{\sigma}\left(\bar{u}_{\sigma}\right) .
$$

Finally, we have the following property

$$
i \in I_{\sigma}^{0}\left(\bar{u}_{\sigma}\right) \Longleftrightarrow\left(\sum_{j=1}^{N_{\tau}} \tau_{j} \bar{\phi}_{i, j}^{2}\right)^{1 / 2} \leq \mu .
$$

Proof The proof follows the lines of that of [10, Th 5.2] with the obvious changes and taking into account formulas (19) and (24).

\section{Convergence and error estimates}

In this section we prove the convergence $\bar{u}_{\sigma} \rightarrow \bar{u}$ and then we establish some error estimates for $\bar{u}_{\sigma}-\bar{u}$. First, we prove some properties of the mesh dependent norms $\|\cdot\|_{\sigma}$ and $|\cdot|_{\sigma}$ and we recall a useful continuity property of Cartensen's quasi-interpolation operator.

Lemma 6.1 The following inequalities hold $\forall u_{\sigma} \in \mathcal{V}_{\sigma}$ :

$$
\begin{aligned}
& \left\|u_{\sigma}\right\|_{L^{2}\left(Q_{h}\right)}^{2} \leq\left\|u_{\sigma}\right\|_{\sigma}^{2}, \\
& \left\|u_{\sigma}\right\|_{L^{1}\left(\Omega_{h} ; L^{2}(0, T)\right)} \leq\left|u_{\sigma}\right|_{\sigma} .
\end{aligned}
$$

Moreover we have that for every $z \in L^{2}(\Omega)$

$$
\left\|\Pi_{h} z\right\|_{L^{2}\left(\Omega_{h}\right)} \leq\|z\|_{L^{2}(\Omega)} .
$$


Proof By the convexity of the real function $g(s)=s^{2}$ and the fact that

$$
\sum_{i=1}^{N_{h}} e_{i}(x)=1 \text { and } 0 \leq e_{i}(x) \leq 1 \quad \forall x \in \Omega_{h}
$$

we get

$$
\begin{aligned}
& \left\|u_{\sigma}\right\|_{L^{2}\left(Q_{h}\right)}^{2}=\int_{\Omega_{h}} \int_{0}^{T}\left(\sum_{i=1}^{N_{h}} \sum_{j=1}^{N_{\tau}} u_{i, j} e_{i} \chi_{j}\right)^{2} d t d x \\
& =\int_{\Omega_{h}} \sum_{j=1}^{N_{\tau}} \tau_{j}\left(\sum_{i=1}^{N_{h}} u_{i, j} e_{i}\right)^{2} d x \leq \sum_{i=1}^{N_{h}} \sum_{j=1}^{N_{\tau}} u_{i, j}^{2} \tau_{j} \int_{\Omega_{h}} e_{i} d x=\left\|u_{\sigma}\right\|_{\sigma}^{2} .
\end{aligned}
$$

To deduce (29) we use the convexity of the norm in $\mathbb{R}^{N_{\tau}}$

$$
|\xi|=\left[\sum_{j=1}^{N_{\tau}} \tau_{j} \xi_{j}^{2}\right]^{1 / 2}
$$

as follows

$$
\begin{aligned}
& \left\|u_{\sigma}\right\|_{L^{1}\left(\Omega_{h} ; L^{2}(0, T)\right)}=\int_{\Omega_{h}}\left[\int_{0}^{T}\left(\sum_{i=1}^{N_{h}} \sum_{j=1}^{N_{\tau}} u_{i, j} e_{i} \chi_{j}\right)^{2} d t\right]^{1 / 2} d x \\
& =\int_{\Omega_{h}}\left[\sum_{j=1}^{N_{\tau}} \tau_{j}\left(\sum_{i=1}^{N_{h}} u_{i, j} e_{i}\right)^{2}\right]^{1 / 2} d x \leq \sum_{i=1}^{N_{h}} \int_{\Omega_{h}} e_{i} d x\left[\sum_{j=1}^{N_{\tau}} \tau_{j} u_{i, j}^{2}\right]^{1 / 2}=\left|u_{\sigma}\right|_{\sigma} .
\end{aligned}
$$

Finally, we prove the inequality (30). Using again the convexity of $g(s)=s^{2}$ and the properties of $\left\{e_{i}\right\}_{i=1}^{N_{h}}$ as above, we get

$$
\begin{gathered}
\left\|\Pi_{h} z\right\|_{L^{2}\left(\Omega_{h}\right)}^{2}=\int_{\Omega_{h}}\left(\sum_{i=1}^{N_{h}} \frac{\int_{\Omega_{h}} z e_{i} d x}{\int_{\Omega_{h}} e_{i} d x} e_{i}\right)^{2} d x \leq \sum_{i=1}^{N_{h}}\left(\frac{\int_{\Omega_{h}} z e_{i} d x}{\int_{\Omega_{h}} e_{i} d x}\right)^{2} \int_{\Omega_{h}} e_{i} d x \\
=\sum_{i=1}^{N_{h}} \frac{\left(\int_{\Omega_{h}} z \sqrt{e_{i}} \sqrt{e_{i}} d x\right)^{2}}{\int_{\Omega_{h}} e_{i} d x} \leq \sum_{i=1}^{N_{h}} \int_{\Omega_{h}} z^{2} e_{i} d x=\|z\|_{L^{2}\left(\Omega_{h}\right)}^{2} \leq\|z\|_{L^{2}(\Omega)}^{2} .
\end{gathered}
$$

Using this properties, we are able to show the boundness of the discrete optimal controls.

Lemma 6.2 Let $\bar{u}_{\sigma} \in \mathcal{Y}_{\sigma}$ be the solution of $\left(\mathrm{P}_{\sigma}\right)$. Then, there exist $C>0$ independent of $\sigma$ such that

$$
\left\|\bar{u}_{\sigma}\right\|_{L^{2}\left(Q_{h}\right)} \leq C
$$


Proof Using (28), we have that

$$
\frac{\nu}{2}\left\|\bar{u}_{\sigma}\right\|_{L^{2}\left(Q_{h}\right)}^{2} \leq \frac{\nu}{2}\left\|\bar{u}_{\sigma}\right\|_{\sigma}^{2} \leq J_{\sigma}\left(\bar{u}_{\sigma}\right) \leq J_{\sigma}(0) .
$$

From the stability estimates in [18, Th 4.6] [20, Th 5.2] we have that there exists a constant independent of $\sigma$ such that

$$
\left\|y_{\sigma}(0)\right\|_{L^{2}(Q)} \leq C\left\|y_{0}\right\|_{L^{2}(\Omega)}
$$

and therefore we can deduce that there exists $C>0$ independent of $\sigma$ such that

$$
\left\|\bar{u}_{\sigma}\right\|_{L^{2}\left(Q_{h}\right)}^{2} \leq C .
$$

Now, given $u \in L^{1}(0, T)$ we set

$$
\pi_{\tau} u=\sum_{j=1}^{N_{\tau}} \frac{1}{\tau_{j}} \int_{t_{j-1}}^{t_{j}} u(t) d t \chi_{j} .
$$

Hence, $\pi_{\tau} u$ is the projection of $u$ on the space of piecewise constant functions and

$$
\left\|\pi_{\tau} u\right\|_{L^{2}(0, T)} \leq\|u\|_{L^{2}(0, T)} \quad \forall u \in L^{2}(0, T) .
$$

Lemma 6.3 Let $u \in L^{1}(Q)$ and set

$$
u_{\sigma}=\pi_{\tau} \Pi_{h} u=\Pi_{h} \pi_{\tau} u=\sum_{i=1}^{N_{h}} \sum_{j=1}^{N_{\tau}}\left(\frac{1}{\tau_{j} \int_{\Omega_{h}} e_{i} d x} \int_{t_{j-1}}^{t_{j}} \int_{\Omega_{h}} u(x, t) e_{i}(x) d x d t\right) e_{i} \chi_{j} .
$$

Then we have

$$
\begin{aligned}
& \left\|u_{\sigma}\right\|_{\sigma} \leq\|u\|_{L^{2}(Q)} \quad \forall u \in L^{2}(Q), \\
& \left|u_{\sigma}\right|_{\sigma} \leq\|u\|_{L^{1}\left(\Omega ; L^{2}(0, T)\right)} \quad \forall u \in L^{1}\left(\Omega ; L^{2}(0, T)\right), \\
& \left\|u_{\sigma}\right\|_{L^{2}\left(Q_{h}\right)} \leq\|u\|_{L^{2}(Q)} \quad \forall u \in L^{2}(Q) . \\
& \left\|u_{\sigma}\right\|_{L^{\infty}\left(Q_{h}\right)} \leq\|u\|_{L^{\infty}(Q)} \quad \forall u \in L^{\infty}(Q) .
\end{aligned}
$$

Moreover, if $u \in L^{2}(Q)$, then the convergence $\left\|u_{\sigma}-u\right\|_{L^{2}\left(Q_{h}\right)} \rightarrow 0$ holds.

Proof Let us prove (33).

$$
\begin{aligned}
& \left\|u_{\sigma}\right\|_{\sigma}^{2}=\sum_{i=1}^{N_{h}} \int_{\Omega_{h}} e_{i} d x \sum_{j=1}^{N_{\tau}} \tau_{j} u_{i, j}^{2} \\
& =\sum_{i=1}^{N_{h}} \int_{\Omega_{h}} e_{i} d x \sum_{j=1}^{N_{\tau}} \tau_{j}\left(\frac{1}{\tau_{j} \int_{\Omega_{h}} e_{i} d x} \int_{\Omega_{h}} \int_{t_{j-1}}^{t_{j}} e_{i}(x) u(x, t) d t d x\right)^{2} \\
& =\sum_{i=1}^{N_{h}} \sum_{j=1}^{N_{\tau}} \frac{1}{\tau_{j} \int_{\Omega_{h}} e_{i} d x}\left(\int_{\Omega_{h}} \int_{t_{j-1}}^{t_{j}} \sqrt{e_{i}(x)} \sqrt{e_{i}(x)} u(x, t) d t d x\right)^{2} \\
& \leq \sum_{i=1}^{N_{h}} \sum_{j=1}^{N_{\tau}} \int_{\Omega_{h}} \int_{t_{j-1}}^{t_{j}} e_{i}(x) u^{2}(x, t) d t d x=\|u\|_{L^{2}\left(Q_{h}\right)}^{2} \leq\|u\|_{L^{2}(Q)}^{2},
\end{aligned}
$$


where we have used (31).

Inequality (34) is proved with (32) as follows

$$
\begin{aligned}
\left|u_{\sigma}\right|_{\sigma} & =\sum_{i=1}^{N_{h}} \int_{\Omega_{h}} e_{i}(x) d x\left[\sum_{j=1}^{N_{\tau}} \tau_{j} u_{i, j}^{2}\right]^{1 / 2} \\
& =\sum_{i=1}^{N_{h}} \int_{\Omega_{h}} e_{i}(x) d x\left[\sum_{j=1}^{N_{\tau}} \tau_{j}\left(\frac{1}{\tau_{j} \int_{\Omega_{h}} e_{i} d x} \int_{\Omega_{h}} \int_{t_{j-1}}^{t_{j}} e_{i}(x) u(x, t) d t d x\right)^{2}\right]^{1 / 2} \\
& =\sum_{i=1}^{N_{h}}\left[\sum_{j=1}^{N_{\tau}} \tau_{j}\left(\int_{\Omega_{h}} \frac{1}{\tau_{j}} \int_{t_{j-1}}^{t_{j}} u(x, t) d t e_{i}(x) d x\right)^{2}\right]^{1 / 2} \\
& =\sum_{i=1}^{N_{h}}\left[\sum_{j=1}^{N_{\tau}} \tau_{j}\left(\int_{\Omega_{h}} \pi_{\tau} u(x, t) e_{i}(x) d x \chi_{j}(t)\right)^{2}\right]^{N_{h}}\left\|\int_{\Omega_{h}} \pi_{\tau} u(x, t) e_{i}(x) d x\right\|_{L^{2}(0, T)} \\
& =\sum_{i=1}^{N_{h}} \int_{\Omega_{h}}\left\|\pi_{\tau} u(x)\right\|_{L^{2}(0, T)} e_{i}(x) d x \\
& \leq \sum_{i=1} \int_{\Omega_{h}}\left\|\pi_{\tau} u(x)\right\|_{L^{2}(0, T)} d x \leq \int_{\Omega_{h}}\|u(x)\|_{L^{2}(0, T)} d x \\
& =\int_{L^{1}\left(\Omega_{h} ; L^{2}(0, T)\right)} \leq\|u\|_{L^{1}\left(\Omega ; L^{2}(0, T)\right)} .
\end{aligned}
$$

The inequalities (35) and (36) are obvious. Finally we prove the convergence of the sequence $\left\{u_{\sigma}\right\}_{\sigma}$. From (30) and the convergence of $\pi_{\tau} u$ and $\Pi_{h} u$ we obtain

$$
\begin{aligned}
\left\|u_{\sigma}-u\right\|_{L^{2}\left(Q_{h}\right)} & \leq\left\|\Pi_{h}\left(\pi_{\tau} u-u\right)\right\|_{L^{2}\left(Q_{h}\right)}+\left\|\Pi_{h} u-u\right\|_{L^{2}\left(Q_{h}\right)} \\
& \leq\left\|\pi_{\tau} u-u\right\|_{L^{2}(Q)}+\left\|\Pi_{h} u-u\right\|_{L^{2}\left(Q_{h}\right)} \rightarrow 0 .
\end{aligned}
$$

Remark 6.4 We can deduce -like in the continuous case, see [8, Remark 2.10], or the case of piecewise constant approximations, see [10, Remark 4]- the existence of a critical value $\mu_{c}>0$ such that $\bar{u}_{\sigma} \equiv 0$ for all $\mu>\mu_{c}$ and all $\sigma$.

Indeed, using the uniform boundness of $\left\{\bar{u}_{\sigma}\right\}$ in $L^{2}\left(Q_{h}\right)$ proved in Lemma 6.2 and applying the standard stability estimates to the discretization of the state equation, we conclude the existence of $C>0$ such that

$$
\left\|\bar{y}_{\sigma}\right\|_{L^{\infty}\left(0, T ; L^{2}(\Omega)\right)}+\left\|\bar{y}_{\sigma}\right\|_{L^{2}\left(0, T ; H_{0}^{1}(\Omega)\right)} \leq C .
$$

Now, arguing as in [21, Theorems 3.1 and 4.1] for $n=2$ and [20] for $n=3$, we deduce the existence of a positive constant, let us call it $\mu_{c}$, that depends on the data on the problem but not on the discretization parameters such that

$$
\left\|\bar{\varphi}_{\sigma}\right\|_{L^{\infty}(Q)} \leq \hat{\mu}_{c}
$$


Finally, using (36) and the continuous embedding $L^{\infty}(0, T) \hookrightarrow L^{2}(0, T)$,

$$
\left\|\Pi_{h} \bar{\varphi}_{\sigma}\right\|_{L^{\infty}\left(\Omega ; L^{2}(0, T)\right)} \leq \sqrt{T} \hat{\mu}_{c}=\mu_{c} .
$$

So, from (27) we deduce that for $\mu \geq \mu_{c}, \bar{u}_{\sigma} \equiv 0$ for all $\sigma$.

Now we can show that the solutions of the discretized problems converge strongly to the solution of problem $(\mathrm{P})$ in $L^{2}(Q)$.

Theorem 6.5 Let $\left(\bar{u}_{\sigma}\right)_{\sigma}$ be a sequence of solutions of problems $\left(\mathrm{P}_{\sigma}\right)$ with $\sigma \rightarrow(0,0)$. Then, the following identities hold

$$
\lim _{\sigma \rightarrow(0,0)} J_{\sigma}\left(\bar{u}_{\sigma}\right)=J(\bar{u})=\inf (\mathrm{P}) \text { and } \lim _{\sigma \rightarrow(0,0)}\left\|\bar{u}_{\sigma}-\bar{u}\right\|_{L^{2}\left(Q_{h}\right)}=0
$$

where $\bar{u}$ is the solution of $(\mathrm{P})$.

Proof Through the proof, we will consider that all the elements of $\mathcal{V}_{\sigma}$ are extended by zero to $Q_{h} \backslash Q$ From Lemma 6.2 we know that $\left\{\bar{u}_{\sigma}\right\}_{\sigma}$ is bounded in $L^{2}(Q)$. Hence, we can extract a subsequence, still denoted in the same way, such that $\bar{u}_{\sigma} \rightarrow u^{*}$ weakly in $L^{2}(Q)$. We are going to prove that $u^{*}=\bar{u}$.

Let $u_{\sigma}=\pi_{\tau} \Pi_{h} \bar{u} \in \mathcal{V}_{\sigma}$. The weak convergence $\bar{u}_{\sigma} \rightarrow u^{*}$ in $L^{2}(Q)$ implies the weak convergence $y_{\bar{u}_{\sigma}} \rightarrow y_{u^{*}}$ in $W(0, T)=L^{2}\left(0, T ; H^{1}(\Omega)\right) \cap H^{1}\left(0, T ; H^{1}(\Omega)^{*}\right)$. From the compactness of the embedding $W(0, T) \subset L^{2}(Q)$, we infer that $y_{\bar{u}_{\sigma}} \rightarrow y_{u^{*}}$ in $L^{2}(Q)$. On the other hand, (14) implies that $y_{\sigma}\left(\bar{u}_{\sigma}\right)-y_{\bar{u}_{\sigma}} \rightarrow 0$ in $L^{2}(Q)$, so we have that $y_{\sigma}\left(\bar{u}_{\sigma}\right) \rightarrow y_{u^{*}}$ in $L^{2}(Q)$. In the same way, the convergence $u_{\sigma} \rightarrow \bar{u}$ in $L^{2}(Q)$ proved in Lemma 6.3 and (14) imply that $y_{\sigma}\left(u_{\sigma}\right) \rightarrow y_{\bar{u}}$ in $L^{2}(Q)$. This, together with (28), (29), (33), and (34) leads to

$$
\begin{aligned}
J\left(u^{*}\right) & \leq \liminf _{\sigma \rightarrow(0,0)}\left(\frac{1}{2}\left\|y_{\sigma}\left(\bar{u}_{\sigma}\right)-y_{d}\right\|_{L^{2}(Q)}^{2}+\frac{\nu}{2}\left\|\bar{u}_{\sigma}\right\|_{L^{2}(Q)}^{2}+\mu j\left(\bar{u}_{\sigma}\right)\right) \\
& \leq \liminf _{\sigma \rightarrow(0,0)} J_{\sigma}\left(\bar{u}_{\sigma}\right) \leq \limsup _{\sigma \rightarrow(0,0)} J_{\sigma}\left(\bar{u}_{\sigma}\right) \leq \limsup _{\sigma \rightarrow(0,0)} J_{\sigma}\left(u_{\sigma}\right) \\
& \leq \limsup _{\sigma \rightarrow(0,0)}\left(\frac{1}{2}\left\|y_{\sigma}\left(u_{\sigma}\right)-y_{d}\right\|_{L^{2}(Q)}^{2}+\frac{\nu}{2}\|\bar{u}\|_{L^{2}(Q)}^{2}+\mu j(\bar{u})\right)=J(\bar{u}) .
\end{aligned}
$$

Since the solution of $(\mathrm{P})$ is unique, then $u^{*}=\bar{u}$, and the whole sequence $\left\{\bar{u}_{\sigma}\right\}_{\sigma}$ converges weakly to $\bar{u}$. Let us prove now the strong convergence. We have just proved that $J_{\sigma}\left(\bar{u}_{\sigma}\right) \rightarrow J(\bar{u})$. This, together with the strong convergence $\bar{y}_{\sigma} \rightarrow \bar{y}$, implies that

$$
\lim _{\sigma \rightarrow(0,0)}\left(\frac{\nu}{2}\left\|\bar{u}_{\sigma}\right\|_{\sigma}^{2}+\mu j_{\sigma}\left(\bar{u}_{\sigma}\right)\right)=\frac{\nu}{2}\|\bar{u}\|_{L^{2}(Q)}^{2}+\mu j(\bar{u})
$$

On the other hand, using the convexity of $j(u)$, the weak convergence $\bar{u}_{\sigma} \rightarrow \bar{u}$ and (29), we have that

$$
j(\bar{u}) \leq \liminf _{\sigma \rightarrow(0,0)} j\left(\bar{u}_{\sigma}\right) \leq \liminf _{\sigma \rightarrow(0,0)} j_{\sigma}\left(\bar{u}_{\sigma}\right) .
$$


Using the weak convergence $\bar{u}_{\sigma} \rightarrow \bar{u}$ in $L^{2}(Q),(28),(38)$ and (39) we have

$$
\begin{aligned}
\frac{\nu}{2}\|\bar{u}\|_{L^{2}(Q)}^{2} & \leq \liminf _{\sigma \rightarrow(0,0)} \frac{\nu}{2}\left\|\bar{u}_{\sigma}\right\|_{L^{2}(Q)}^{2} \leq \limsup _{\sigma \rightarrow(0,0)} \frac{\nu}{2}\left\|\bar{u}_{\sigma}\right\|_{L^{2}(Q)}^{2} \leq \limsup _{\sigma \rightarrow(0,0)} \frac{\nu}{2}\left\|\bar{u}_{\sigma}\right\|_{\sigma}^{2} \\
& \leq \limsup _{\sigma \rightarrow(0,0)}\left(\frac{\nu}{2}\left\|\bar{u}_{\sigma}\right\|_{\sigma}^{2}+\mu j_{\sigma}\left(\bar{u}_{\sigma}\right)\right)-\liminf _{\sigma \rightarrow(0,0)} \mu j_{\sigma}\left(\bar{u}_{\sigma}\right) \\
& \leq \frac{\nu}{2}\|\bar{u}\|_{L^{2}(Q)}^{2}+\mu j(\bar{u})-\mu j(\bar{u})=\frac{\nu}{2}\|\bar{u}\|_{L^{2}(Q)}^{2}
\end{aligned}
$$

from where we readily deduce the strong convergence in $L^{2}(Q)$.

\subsection{Error Estimates}

We will establish now the error estimates for Cartensen quasi-interpolation operator as well as for the projection operator.

Lemma 6.6 There exists $C>0$ independent of $h$ and $\tau$ such that

$$
\begin{aligned}
h\left\|u-\Pi_{h} u\right\|_{L^{2}\left(\Omega_{h}\right)}+\left\|u-\Pi_{h} u\right\|_{H^{1}\left(\Omega_{h}\right)^{*}} & \leq C h^{2}\|u\|_{H^{1}(\Omega)} \forall u \in H^{1}(\Omega), \\
\tau\left\|u-\pi_{\tau} u\right\|_{L^{2}(0, T)}+\left\|u-\pi_{\tau} u\right\|_{H^{1}(0, T)^{*}} & \leq C \tau^{2}\|u\|_{H^{1}(0, T)} \forall u \in H^{1}(0, T) .
\end{aligned}
$$

Moreover, for every $u \in H^{1}(Q)$,

$$
\begin{aligned}
\left\|u-u_{\sigma}\right\|_{L^{2}\left(0, T ; H^{1}\left(\Omega_{h}\right)^{*}\right)} & \leq C\left(\tau+h^{2}\right)\|u\|_{H^{1}(Q)}, \\
\left\|u-u_{\sigma}\right\|_{L^{2}\left(Q_{h}\right)} & \leq C(\tau+h)\|u\|_{H^{1}(Q)}, \\
\left\|u-u_{\sigma}\right\|_{H^{1}(Q)^{*}} & \leq C\left(\tau^{2}+h^{2}\right)\|u\|_{H^{1}(Q)}
\end{aligned}
$$

where $u_{\sigma}=\pi_{\tau} \Pi_{h} u$ in $Q_{h}$ and we extend $u_{\sigma}=u$ in $Q \backslash Q_{h}$ in the last inequality.

Proof Estimate (40) follows from the results in [3]; see also [25, Lemma 4.5]. Estimate $(41)$ is the usual estimate for the $L^{2}(0, T)$-projection. Let us prove (42). To this end we use (40), (30) and (41)

$$
\begin{aligned}
& \left\|u-u_{\sigma}\right\|_{L^{2}\left(0, T ; H^{1}\left(\Omega_{h}\right)^{*}\right)} \\
& \leq\left\|u-\Pi_{h} u\right\|_{L^{2}\left(0, T ; H^{1}\left(\Omega_{h}\right)^{*}\right)}+\left\|\Pi_{h} u-\Pi_{h} \pi_{\tau} u\right\|_{L^{2}\left(0, T ; H^{1}\left(\Omega_{h}\right)^{*}\right)} \\
& \leq\left(\int_{0}^{T}\left\|u-\Pi_{h} u\right\|_{H^{1}\left(\Omega_{h}\right)^{*}}^{2} d t\right)^{1 / 2}+\left\|\Pi_{h}\left(u-\pi_{\tau} u\right)\right\|_{L^{2}(Q)} \\
& \leq C h^{2}\|u\|_{L^{2}\left(0, T ; H^{1}\left(\Omega_{h}\right)\right)}+\left\|u-\pi_{\tau} u\right\|_{L^{2}\left(Q_{h}\right)} \\
& \leq C h^{2}\|u\|_{L^{2}\left(0, T ; H^{1}(\Omega)\right)}+C \tau\|u\|_{L^{2}\left(\Omega_{h} ; H^{1}(0, T)\right)} \leq C\left(h^{2}+\tau\right)\|u\|_{H^{1}(Q)} .
\end{aligned}
$$
that

To prove (43), we deduce from (41), the fact that $\pi_{\tau}$ is a projection and (40)

$$
\begin{aligned}
\left\|u-u_{\sigma}\right\|_{L^{2}\left(Q_{h}\right)} & \leq\left\|u-\pi_{\tau} u\right\|_{L^{2}\left(Q_{h}\right)}+\left\|\pi_{\tau} u-\pi_{\tau} \Pi_{h} u\right\|_{L^{2}\left(Q_{h}\right)} \\
& \leq C(\tau+h)\|u\|_{H^{1}(Q)} .
\end{aligned}
$$


To prove relation (44), we use the continuity of the embeddings $L^{2}\left(\Omega ; H^{1}(0, T)^{*}\right) \hookrightarrow H^{1}(Q)^{*}$ and $L^{2}\left(0, T ; H^{1}(\Omega)^{*}\right) \hookrightarrow H^{1}(Q)^{*}$, estimate $(41)$, the fact that $\pi_{\tau}$ is a projection in $L^{2}(0, T)$ and $(40)$.

$$
\begin{aligned}
\left\|u-u_{\sigma}\right\|_{H^{1}(Q)^{*}} & \leq\left\|u-\pi_{\tau} u\right\|_{H^{1}(Q)^{*}}+\left\|\pi_{\tau} u-u_{\sigma}\right\|_{H^{1}(Q)^{*}} \\
& \leq\left\|u-\pi_{\tau} u\right\|_{L^{2}\left(\Omega ; H^{1}(0, T)^{*}\right.}+\left\|\pi_{\tau}\left(u-\Pi_{h} u\right)\right\|_{L^{2}\left(0, T ; H^{1}(\Omega)^{*}\right)} \\
& \leq C \tau^{2}\|u\|_{L^{2}\left(\Omega ; H^{1}(0, T)\right)}+C h^{2}\|u\|_{L^{2}\left(0, T ; H^{1}(\Omega)\right)} \\
& \leq C\left(h^{2}+\tau^{2}\right)\|u\|_{H^{1}(Q)}
\end{aligned}
$$

In the following theorem we will extend the elements of $\mathcal{U}_{\sigma}$ by $\bar{u}$ in $Q \backslash Q_{h}$. Notice that, if $\mu>0$, using the sparsity property of the control (10) and the zero boundary condition of the adjoint state equation, we have that for $h>0$ small enough, $\bar{u}=0$ in $Q \backslash Q_{h}$.

Theorem 6.7 Let $\bar{u}$ be the solution of $(\mathrm{P})$ and let $\bar{u}_{\sigma}$ be the solution of $\left(\mathrm{P}_{\sigma}\right)$. Then, there exists $C>0$ independent of $\sigma$ such that for all $h<h_{0}$ and $\tau<\tau_{0}$,

$$
\left\|\bar{u}_{\sigma}-\bar{u}\right\|_{L^{2}(Q)} \leq C(\tau+h) .
$$

Proof Let $y_{\bar{u}_{\sigma}}$ and $\varphi_{\bar{u}_{\sigma}}$ be the continuous state and adjoint state related to the discrete optimal control $\bar{u}_{\sigma}$, defined according to (1) and (4) and $\bar{y}$ and $\bar{\varphi}$ the state and adjoint state related to the optimal control $\bar{u}$, as defined in (7) and (8). Using integration by parts and taking into account the zero boundary conditions for the state and the adjoint state, the equalities $y_{\bar{u}_{\sigma}}(0)=\bar{y}(0)=y_{0}$, and the zero final conditions for the adjoint states, it follows in a standard way that

$$
\begin{aligned}
\left\|y \bar{u}_{\sigma}-\bar{y}\right\|_{L^{2}(Q)}^{2} & =\left(y_{\bar{u}_{\sigma}}-y_{d}-\left(\bar{y}-y_{d}\right), y_{\bar{u}_{\sigma}}-\bar{y}\right)_{Q} \\
& =\left(\left(-\partial_{t}+A^{*}\right)\left(\varphi_{\bar{u}_{\sigma}}-\bar{\varphi}\right), y_{\bar{u}_{\sigma}}-\bar{y}\right)_{Q} \\
& =\left(\varphi_{\bar{u}_{\sigma}}-\bar{\varphi},\left(\partial_{t}+A\right)\left(y_{\bar{u}_{\sigma}}-\bar{y}\right)\right)_{Q} \\
& =\left(\varphi \bar{u}_{\sigma}-\bar{\varphi},\left(f+\bar{u}_{\sigma}\right)-(f+\bar{u})\right)_{Q} \\
& =\left(\varphi_{\bar{u}_{\sigma}}-\bar{\varphi}, \bar{u}_{\sigma}-\bar{u}\right)_{Q}
\end{aligned}
$$

and hence, from (3) we readily deduce that

$$
\left\|y \bar{u}_{\sigma}-\bar{y}\right\|_{L^{2}(Q)}^{2}+\nu\left\|\bar{u}-\bar{u}_{\sigma}\right\|_{L^{2}(Q)}^{2}=F^{\prime}\left(\bar{u}_{\sigma}\right)\left(\bar{u}_{\sigma}-\bar{u}\right)-F^{\prime}(\bar{u})\left(\bar{u}_{\sigma}-\bar{u}\right) .
$$

Let us denote now $u_{\sigma}=\pi_{\tau} \Pi_{h} \bar{u}$. From the first order optimality conditions for $(\mathrm{P})$ and $\left(\mathrm{P}_{\sigma}\right)$ and the definition of subdifferential, we have

$$
\begin{gathered}
F^{\prime}(\bar{u})\left(\bar{u}_{\sigma}-\bar{u}\right)+\mu j\left(\bar{u}_{\sigma}\right)-\mu j(\bar{u}) \geq 0, \\
F_{\sigma}^{\prime}\left(\bar{u}_{\sigma}\right)\left(u_{\sigma}-\bar{u}_{\sigma}\right)+\mu j_{\sigma}\left(u_{\sigma}\right)-\mu j_{\sigma}\left(\bar{u}_{\sigma}\right) \geq 0 .
\end{gathered}
$$

Adding this inequalities and taking into account that $j\left(\bar{u}_{\sigma}\right) \leq j_{\sigma}\left(\bar{u}_{\sigma}\right)$, see $(29)$, and $j_{\sigma}\left(u_{\sigma}\right) \leq j(\bar{u})$, see $(34)$, we obtain

$$
F^{\prime}(\bar{u})\left(\bar{u}_{\sigma}-\bar{u}\right)+F_{\sigma}^{\prime}\left(\bar{u}_{\sigma}\right)\left(u_{\sigma}-\bar{u}_{\sigma}\right) \geq 0 .
$$


From the previous inequality and (46) we have that

$$
\begin{aligned}
\nu\left\|\bar{u}-\bar{u}_{\sigma}\right\|_{L^{2}(Q) \leq}^{2} & F_{\sigma}^{\prime}\left(\bar{u}_{\sigma}\right)\left(u_{\sigma}-\bar{u}_{\sigma}\right)+F^{\prime}\left(\bar{u}_{\sigma}\right)\left(\bar{u}_{\sigma}-\bar{u}\right) \\
\leq & F_{\sigma}^{\prime}\left(\bar{u}_{\sigma}\right)\left(u_{\sigma}-\bar{u}_{\sigma}\right)-F^{\prime}\left(\bar{u}_{\sigma}\right)\left(u_{\sigma}-\bar{u}_{\sigma}\right)+F^{\prime}\left(\bar{u}_{\sigma}\right)\left(u_{\sigma}-\bar{u}\right) \\
= & \int_{Q}\left(\bar{\varphi}_{\sigma}-\varphi_{\bar{u}_{\sigma}}\right)\left(u_{\sigma}-\bar{u}_{\sigma}\right) d x d t+\nu\left(\bar{u}_{\sigma}, u_{\sigma}-\bar{u}_{\sigma}\right)_{\sigma} \\
& -\nu \int_{Q} \bar{u}_{\sigma}\left(u_{\sigma}-\bar{u}_{\sigma}\right) d x d t+F^{\prime}\left(\bar{u}_{\sigma}\right)\left(u_{\sigma}-\bar{u}\right)
\end{aligned}
$$

The first term is estimated with

$$
\begin{aligned}
& \int_{Q}\left(\bar{\varphi}_{\sigma}-\varphi_{\bar{u}_{\sigma}}\right)\left(u_{\sigma}-\bar{u}_{\sigma}\right) d x d t= \\
& \int_{Q}\left(\bar{\varphi}_{\sigma}-\varphi_{\bar{u}_{\sigma}}\right)\left(u_{\sigma}-\bar{u}\right) d x d t+\int_{Q}\left(\bar{\varphi}_{\sigma}-\varphi_{\bar{u}_{\sigma}}\right)\left(\bar{u}-\bar{u}_{\sigma}\right) d x d t \\
& \leq\left\|\bar{\varphi}_{\sigma}-\varphi_{\bar{u}_{\sigma}}\right\|_{L^{2}(Q)}\left\|u_{\sigma}-\bar{u}\right\|_{L^{2}(Q)}+\left\|\bar{\varphi}_{\sigma}-\varphi_{\bar{u}_{\sigma}}\right\|_{L^{2}(Q)}\left\|\bar{u}-\bar{u}_{\sigma}\right\|_{L^{2}(Q)} \\
& \leq C\left(\tau+h^{2}\right)\left\|\bar{u}_{\sigma}\right\|_{L^{2}(Q)}\left[(\tau+h)\|\bar{u}\|_{H^{1}(Q)}+\left\|\bar{u}-\bar{u}_{\sigma}\right\|_{L^{2}(Q)}\right] \\
& \leq C(\tau+h)^{2}+C(\tau+h)\left\|\bar{u}-\bar{u}_{\sigma}\right\|_{L^{2}(Q)}
\end{aligned}
$$

where we can use the finite element error estimates in $[18,20]$ thanks to the stability estimate in Lemma 6.2 and (43) thanks to the regularity of the optimal control stated in Theorem 3.2.

The next two terms satisfy

$$
\begin{aligned}
& \nu\left(\bar{u}_{\sigma}, u_{\sigma}-\bar{u}_{\sigma}\right)_{\sigma}-\nu \int_{Q} \bar{u}_{\sigma}\left(u_{\sigma}-\bar{u}_{\sigma}\right) d x d t \\
& =\nu\left(\bar{u}_{\sigma}, u_{\sigma}-\bar{u}_{\sigma}\right)_{\sigma}-\nu \int_{Q} \bar{u}_{\sigma}\left(u_{\sigma}-\bar{u}\right) d x d t-\nu \int_{Q} \bar{u}_{\sigma}\left(\bar{u}-\bar{u}_{\sigma}\right) d x d t \\
& \nu\left(\bar{u}_{\sigma}, u_{\sigma}\right)_{\sigma}-\nu\left\|\bar{u}_{\sigma}\right\|_{\sigma}^{2}-\nu \int_{Q} \bar{u}_{\sigma}\left(u_{\sigma}-\bar{u}\right) d x d t-\nu \int_{Q} \bar{u}_{\sigma} \bar{u} d x d t+\nu\left\|\bar{u}_{\sigma}\right\|_{L^{2}(Q)}^{2} \\
& \leq \nu \int_{Q} \bar{u}_{\sigma}\left(\bar{u}-u_{\sigma}\right) d x d t
\end{aligned}
$$

where we have used (28) and that $u_{\sigma}=\pi_{\tau} \Pi_{h} \bar{u}$ together with the fact that $\pi_{\tau}$ is a projection in $L^{2}(0, T)$ and the projection-like formula for Carstensen's quasiinterpolation (17) to deduce that $\left(\bar{u}_{\sigma}, u_{\sigma}\right)_{\sigma}=\int_{Q} \bar{u}_{\sigma} \bar{u} d x d t$.

To estimate the last term in the right hand side of (47) we write

$$
\begin{aligned}
& F^{\prime}\left(\bar{u}_{\sigma}\right)\left(u_{\sigma}-\bar{u}\right)=F^{\prime}\left(\bar{u}_{\sigma}\right)\left(u_{\sigma}-\bar{u}\right)-F^{\prime}(\bar{u})\left(u_{\sigma}-\bar{u}\right)+F^{\prime}(\bar{u})\left(u_{\sigma}-\bar{u}\right) \\
& =\int_{Q}\left(\varphi_{\bar{u}_{\sigma}}-\bar{\varphi}\right)\left(u_{\sigma}-\bar{u}\right) d x d t+\nu \int_{Q} \bar{u}_{\sigma}\left(u_{\sigma}-\bar{u}\right) d x d t+\int_{Q} \bar{\varphi}\left(u_{\sigma}-\bar{u}\right) d x d t \\
& \leq\left\|\varphi_{\bar{u}_{\sigma}}-\bar{\varphi}\right\|_{L^{2}(Q)}\left\|u_{\sigma}-\bar{u}\right\|_{L^{2}(Q)}+\nu \int_{Q} \bar{u}_{\sigma}\left(u_{\sigma}-\bar{u}\right) d x d t \\
& +\|\bar{\varphi}\|_{H^{1}(Q)}\left\|u_{\sigma}-\bar{u}\right\|_{H^{1}(Q)^{*}} \\
& \leq C\left\|\bar{u}_{\sigma}-\bar{u}\right\|_{L^{2}(Q)}(h+\tau)+\nu \int_{Q} \bar{u}_{\sigma}\left(u_{\sigma}-\bar{u}\right) d x d t+C\left(h^{2}+\tau^{2}\right)\|\bar{u}\|_{H^{1}(Q)},
\end{aligned}
$$


where we have used the Lipschitz dependance of the adjoint state with respect to the control (the dependance is linear continuous indeed), (43), (44) and the $H^{1}(Q)$ regularity of the optimal control and its related adjoint state provided in Theorem 3.2 .

And the proof follows collecting all the estimates.

\section{Some extensions}

In [10] we investigated the approximation of the controls by means of piecewise constant functions in both space and time. We obtained an order of $\mathcal{O}(\sqrt{\tau}+h)$ for a problem governed by a semilinear parabolic equation. The proofs of Theorem 5.7 of that reference can be adapted to the approximation shown in the work at hand to obtain the same order of convergence for a problem with a semilinear state equation.

Conversely, the proof of Theorem 6.7 can be adapted to the case of using piecewise constant approximations for the control to obtain also $\mathcal{O}(\tau+h)$ for a problem governed by a linear equation. The main difference is that we should take the $L^{2}(\Omega)$ projection $\pi_{h}$ instead of Carstensen's quasi-interpolation, and the proof can be slightly simplified: there is no need for numerical integration in the computation of the norms that appear in the objective functional, the terms that appear in the left had side of (49) are zero and so is the midterm in the right hand side of $(50)$.

\section{Numerical experiments}

We report on three numerical experiments. In the first one, we describe an example with known solution and show error estimates (cf. Theorem 6.7 and [10, Theorem 5.7]). In the second one, we show how the sparsity properties of the solution change as $\mu$ changes (cf. Remark 6.4 and [8, Remark 2.10]). In the third one, we show the sparsity pattern for a $2 \mathrm{D}$ example.

8.1 Experiment 1. Error estimates for an example with known solution.

Let $\Omega=(0,1) \subset \mathbb{R}$ and $T=1$. We are going to consider the data of the model example in [10, Section 6.1], where $y_{0}=0, f=0, A=-\partial_{x x}^{2}, \nu=1, \mu=0.1$, and $\bar{u}(x, t)=\sqrt{2} \chi_{(0.25,0.75)}(x)(x-0.25)(x-0.75) \sin (2 \pi t)$ is the optimal control for some $y_{d}$ conveniently chosen.

We have solved the problem using approximations of the control in $\mathcal{V}_{\sigma}$, the space of continuous piecewise linear functions in space described in this work,

$$
\mathcal{V}_{\sigma}=\left\{u_{\sigma} \in L^{2}\left(0, T ; V_{h}\right): u_{\sigma \mid I_{j}} \in V_{h} \forall j=1, \ldots, N_{\tau}\right\},
$$

where

$$
V_{h}=\left\{u_{h} \in C\left(\bar{\Omega}_{h}\right): z_{h \mid K} \in P_{1}(K) \quad \forall K \in \mathcal{K}_{h}\right\} .
$$

For easier comparison, we also include the results provided in [10] using piecewise constant control functions.

$$
\mathcal{U}_{\sigma}=\left\{u_{\sigma} \in L^{2}\left(0, T ; U_{h}\right): u_{\sigma \mid I_{j}} \in U_{h} \forall j=1, \ldots, N_{\tau}\right\},
$$


where

$$
U_{h}=\left\{v_{h} \in L^{2}\left(\Omega_{h}\right): u_{h \mid K} \in P_{0}(K) \forall K \in \mathcal{K}_{h}\right\} .
$$

In both cases, we confirm the estimates

$$
\left\|\bar{u}-\bar{u}_{\sigma}\right\|_{L^{2}(Q)}=O(\tau+h) .
$$

We take two families of uniform partitions in space and time, with $h=2^{-i}$, $i=i_{0}, \ldots, I$, and $\tau=2^{-j} j=j_{0}, \ldots, J$ for some values of $I$ and $J$ big enough. We have been able to achieve $I=J=13$ in a PC with MATLAB. To solve the discrete problems, we use a semismooth Newton method as described in [15].

We perform three tests:

1. We take $h=h_{i}$ and $\tau=\tau_{i}$, this is, we let both parameter tend to 0 at the same time.

2. We fix a small $\tau=\tau_{J}$ and take $h=h_{i}$ for $i=i_{0}, \ldots, I^{*}$. This is, we refine only in space.

3. We fix a small $h=h_{I}$ and take $\tau=\tau_{j}$ for $j=j_{0}, \ldots, J^{*}$, so we refine only in time.

To measure the error, we compute

$$
e_{\sigma}=\left\|\bar{u}_{\sigma}-\tilde{\pi}_{\sigma} \bar{u}\right\|_{L^{2}(Q)}
$$

where $\tilde{\pi}_{\sigma} \bar{u}=\tilde{\pi}_{\tau} \tilde{\pi}_{h} \bar{u}$. The operator $\tilde{\pi}_{\tau}$ is the numerical approximation of the $L^{2}(0, T)$ projection onto the set of piecewise constant functions given by the midpoint rule: $\tilde{\pi}_{\tau} f=\sum_{j=1}^{N_{\tau}} f\left(\left(t_{j-1}+t_{j}\right) / 2\right) \chi_{\left(t_{j-1}, t_{j}\right)}$. The operator $\tilde{\pi}_{h}$ is the usual nodal interpolation in space for the experiment with continuous piecewise linear functions in space and $\tilde{\pi}_{h}$ is the numerical approximation of the $L^{2}(\Omega)$ projection onto the set of piecewise constant functions given by the midpoint rule. Let us denote $\sigma_{i, j}=\left(h_{i}, \tau_{j}\right)$. The experimental order of convergence is measured as

$$
E O C_{i}=\frac{\log \left(e_{\sigma_{i, i}}\right)-\log \left(e_{\sigma_{i-1, i-1}}\right)}{\log \left(h_{i}\right)-\log \left(h_{i-1}\right)}
$$

in the first cases and analogously in the other cases.

For the first test $(h=\tau)$, we obtain the results shown in Table 1. The results are as expected from Theorem 6.7.

\begin{tabular}{c|cc||cc} 
& \multicolumn{2}{|c|}{$V_{\sigma}$} & \multicolumn{2}{c}{$U_{\sigma}$} \\
$i$ & $e_{i}$ & $E O C_{i}$ & $e_{i}$ & $E O C_{i}$ \\
\hline 6 & $4.65 \mathrm{E}-3$ & - & $4.37 \mathrm{E}-3$ & - \\
7 & $2.37 \mathrm{E}-3$ & 0.97 & $2.22 \mathrm{E}-3$ & 0.98 \\
8 & $1.20 \mathrm{E}-3$ & 0.99 & $1.12 \mathrm{E}-3$ & 0.99 \\
9 & $6.01 \mathrm{E}-4$ & 0.99 & $5.60 \mathrm{E}-4$ & 0.99 \\
10 & $3.01 \mathrm{E}-4$ & 1.00 & $2.81 \mathrm{E}-4$ & 1.00 \\
11 & $1.51 \mathrm{E}-4$ & 1.00 & $1.40 \mathrm{E}-4$ & 1.00 \\
12 & $7.54 \mathrm{E}-5$ & 1.00 & $7.03 \mathrm{E}-5$ & 1.00 \\
13 & $3.77 \mathrm{E}-5$ & 1.00 & $3.51 \mathrm{E}-5$ & 1.00
\end{tabular}

Table 1 Results for $h_{i}=\tau_{i}=2^{-i}$. Left, continuous piecewise linear functions in space. Right, piecewise constant functions in space. 


\begin{tabular}{c|cc||cc} 
& \multicolumn{3}{|c|}{$V_{\sigma}$} & \multicolumn{2}{c}{$U_{\sigma}$} \\
$i$ & $e_{i}$ & $E O C_{i}$ & $e_{i}$ & $E O C_{i}$ \\
\hline 6 & $2.91 \mathrm{E}-3$ & - & $2.99 \mathrm{E}-3$ & - \\
7 & $1.47 \mathrm{E}-3$ & 0.99 & $1.48 \mathrm{E}-3$ & 1.01 \\
8 & $7.41 \mathrm{E}-4$ & 0.98 & $7.44 \mathrm{E}-4$ & 1.00 \\
9 & $3.78 \mathrm{E}-4$ & 0.97 & $3.76 \mathrm{E}-4$ & 0.98 \\
10 & $1.97 \mathrm{E}-4$ & 0.94 & $1.94 \mathrm{E}-4$ & 0.96 \\
11 & $1.06 \mathrm{E}-4$ & 0.89 & $1.03 \mathrm{E}-4$ & 0.91 \\
12 & $6.04 \mathrm{E}-5$ & 0.81 & $5.75 \mathrm{E}-5$ & 0.84 \\
13 & $3.77 \mathrm{E}-5$ & 0.68 & $3.51 \mathrm{E}-5$ & 0.71
\end{tabular}

Table 2 Results for $\tau=2^{-13}$ and $h_{i}=2^{-i}$. Left, continuous piecewise linear functions in space. Right, piecewise constant functions in space.

For the second test ( $\tau$ fixed and small, refinements only in the space step), we get the results summarized in Table 2. The error due to $\tau=2^{-13}$ is small, but not zero. So the values obtained for the error due to the discretization in space are not of the form $C h_{i}$, but of the form $C h_{i} \pm E_{\tau_{J}}$. So it seems reasonable to discard the results for which the error in time starts to be big enough. For $i \geq 10$ it maybe more than $10 \%$ of the error, so we stop at $I^{*}=9$. We obtain an order of convergence of $O(h)$, as expected.

In Table 3 we show the results for the third test ( $h$ fixed and small, refinements in the time step). Since the spatial error is not zero, we discard the results for which

\begin{tabular}{|c|c|c|c|c|c|}
\hline \multirow[b]{2}{*}{$j$} & \multicolumn{2}{|c|}{$V_{\sigma}$} & \multicolumn{3}{|l|}{$U_{\sigma}$} \\
\hline & $e_{j}$ & $E O C_{j}$ & $e_{j}$ & $E O C_{j}$ & \\
\hline 6 & $1.89 \mathrm{E}-3$ & - & $1.71 \mathrm{E}-3$ & - & \\
\hline 7 & $9.80 \mathrm{E}-4$ & 0.95 & $8.84 \mathrm{E}-4$ & 0.95 & \\
\hline 8 & $5.07 \mathrm{E}-4$ & 0.95 & $4.57 \mathrm{E}-4$ & 0.95 & $*$ \\
\hline 9 & $2.66 \mathrm{E}-4$ & 0.93 & $2.40 \mathrm{E}-4$ & 0.93 & \\
\hline 10 & $1.44 \mathrm{E}-4$ & 0.88 & $1.30 \mathrm{E}-4$ & 0.88 & \\
\hline 11 & $8.34 \mathrm{E}-5$ & 0.79 & $7.54 \mathrm{E}-5$ & 0.79 & \\
\hline 12 & $5.29 \mathrm{E}-5$ & 0.66 & $4.83 \mathrm{E}-5$ & 0.64 & \\
\hline 13 & $3.77 \mathrm{E}-5$ & 0.49 & $3.51 \mathrm{E}-5$ & 0.46 & \\
\hline
\end{tabular}

Table 3 Results for $h=2^{-13}$ and $\tau_{j}=2^{-j}$. Left, continuous piecewise linear functions in space. Right, piecewise constant functions in space.

it is at least the $10 \%$ of the global error and stop at $J^{*}=8$. We obtain an order of convergence close to $O(\tau)$.

\subsection{Experiment 2. Directional sparsity properties of the control}

Let $\Omega=(0,1) \subset \mathbb{R}$ and let $T=1$. We have solved the unconstrained version of the example shown in [8, Remark 2.11]. The data for the example are $y_{0}=0, f=0$, $A=-\partial_{x x}^{2}, \nu=1 e-4, \mu=\mu_{0}=4 e-3$ and

$$
y_{d}(x, t)=\exp \left(-20\left[(x-0.2)^{2}+(t-0.2)^{2}\right]\right)+\exp \left(-20\left[(x-0.7)^{2}+(t-0.9)^{2}\right]\right) .
$$

We solve the problem in a mesh with $h=\tau=2^{-8}$. In Figure 1, we show the support of the optimal control for the values $\mu=M \mu_{0}, M=0, \ldots, 8$. For $\mu=0$, we have 
no sparsity pattern for the control. Then we see how the control is directionally sparse for $\mu>0$ and how the support of the control is smaller as $\mu$ increases. After a few essays, we find that $\bar{u} \equiv 0$ for $\mu \geq 7.4803 \mu_{0}$. As expected, the value of the objective functional increases as $\mu$ increases. You may find the obtained numerical values for $J_{\sigma}\left(\bar{u}_{\sigma}\right)$ in Table 4 .
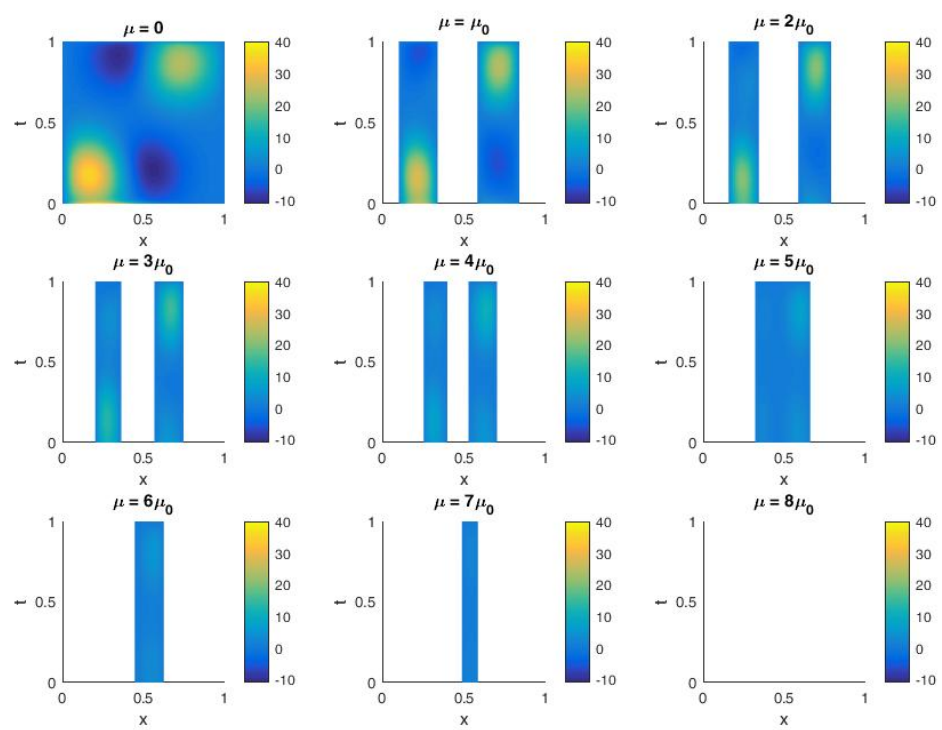

Fig. 1 Experiment 2. Support of the optimal control for different values of $\mu$

\begin{tabular}{c|rrrrr}
$\mu$ & 0 & $\mu_{0}$ & $2 \mu_{0}$ & $3 \mu_{0}$ & $4 \mu_{0}$ \\
\hline$J_{\sigma}\left(\bar{u}_{\sigma}\right)$ & 0.00915 & 0.03410 & 0.04811 & 0.05673 & 0.06215 \\
$\mu$ & $5 \mu_{0}$ & $6 \mu_{0}$ & $7 \mu_{0}$ & $8 \mu_{0}$ & \\
\hline$J_{\sigma}\left(\bar{u}_{\sigma}\right)$ & 0.06550 & 0.06746 & 0.06836 & 0.06847
\end{tabular}

Table 4 Experiment 2. Value of the objective functional as the parameter $\mu$ increases

8.3 Experiment 3. Directional sparsity properties of the control in 2D

Let $\Omega=(0,1) \times(0,1) \subset \mathbb{R}^{2}$ and let $T=1$. We solve a $2 \mathrm{D}$ version of the previous example. The data for the example are $y_{0}=0, f=0, A=-\Delta, \nu=1 e-4$, 
$\mu=\mu_{0}=4 e-3$ and

$$
\begin{aligned}
y_{d}(x, t)= & \exp \left(-20\left[\left(x_{1}-0.2\right)^{2}+\left(x_{2}-0.2\right)^{2}+(t-0.2)^{2}\right]\right) \\
& +\exp \left(-20\left[\left(x_{1}-0.7\right)^{2}+\left(x_{2}-0.7\right)^{2}+(t-0.9)^{2}\right]\right) .
\end{aligned}
$$

We solve the problem in a mesh with $h=\tau=2^{-8}$. In Figures 2 and 3 we see graphs of the approximations obtained using piecewise constant and piecewise linear function respectively in different moments of time.

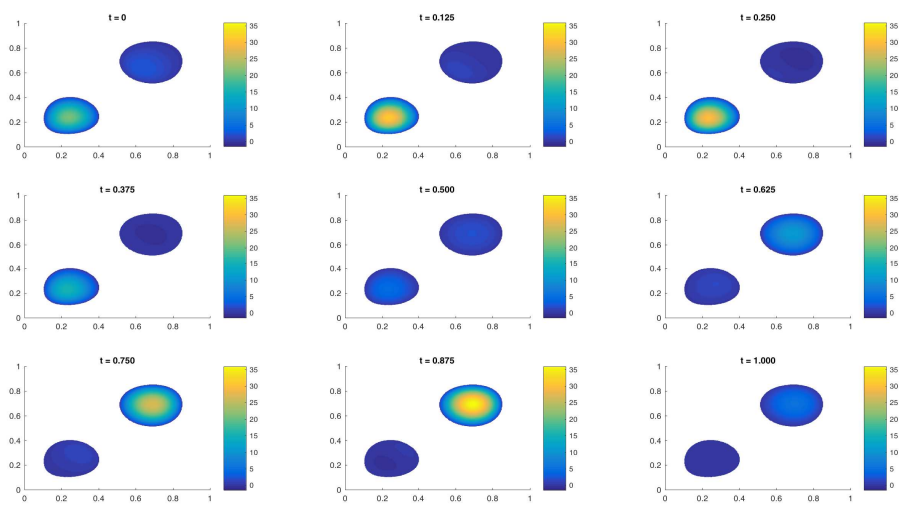

Fig. 2 Experiment 3. Support of the optimal 2D control at different moments of time. Piecewise constant functions. $J_{\sigma}\left(\bar{u}_{\sigma}\right)=0.01565574$
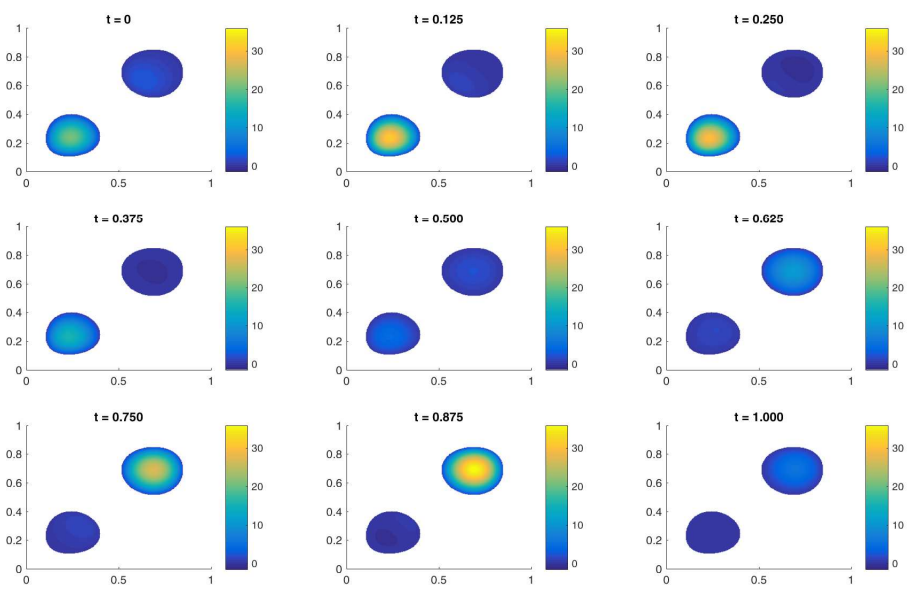

Fig. 3 Experiment 3. Support of the optimal 2D control at different moments of time. Continuous piecewise linear functions. $J_{\sigma}\left(\bar{u}_{\sigma}\right)=0.01565603$ 


\section{References}

1. Boulanger, A.C., Trautmann, P.: Sparse Optimal Control of the KdV-Burgers Equation on a Bounded Domain. SIAM J. Control Optim. 55(6), 3673-3706 (2017). URL https: //doi.org/10.1137/15M1020745

2. Brenner, S.C., Scott, L.R.: The mathematical theory of finite element methods, Texts in Applied Mathematics, vol. 15, second edn. Springer-Verlag, New York (2002). DOI 10.1007/978-1-4757-3658-8. URL http://dx.doi.org/10.1007/978-1-4757-3658-8

3. Carstensen, C.: Quasi-interpolation and a posteriori error analysis in finite element methods. M2AN Math. Model. Numer. Anal. 33(6), 1187-1202 (1999). DOI 10.1051/m2an: 1999140. URL http://dx.doi.org/10.1051/m2an: 1999140

4. Casas, E., Clason, C., Kunisch, K.: Approximation of elliptic control problems in measure spaces with sparse solutions. SIAM J. Control Optim. 50(4), 1735-1752 (2012). DOI 10.1137/110843216. URL http://dx.doi.org/10.1137/110843216

5. Casas, E., Clason, C., Kunisch, K.: Parabolic control problems in measure spaces with sparse solutions. SIAM J. Control Optim. 51(1), 28-63 (2013). DOI 10.1137/120872395. URL http://dx.doi.org/10.1137/120872395

6. Casas, E., Herzog, R., Wachsmuth, G.: Approximation of sparse controls in semilinear equations by piecewise linear functions. Numer. Math. 122(4), 645-669 (2012). DOI 10.1007/s00211-012-0475-7. URL http://dx.doi.org/10.1007/s00211-012-0475-7

7. Casas, E., Herzog, R., Wachsmuth, G.: Optimality conditions and error analysis of semilinear elliptic control problems with $L^{1}$ cost functional. SIAM J. Optim. 22(3), 795-820 (2012). DOI 10.1137/110834366. URL http://dx.doi.org/10.1137/110834366

8. Casas, E., Herzog, R., Wachsmuth, G.: Analysis of spatio-temporally sparse optimal control problems of semilinear parabolic equations. ESAIM Control Optim. Calc. Var. 23(1), 263-295 (2017). DOI 10.1051/cocv/2015048. URL http://dx.doi.org/10.1051/cocv/ 2015048

9. Casas, E., Kunisch, K.: Parabolic control problems in space-time measure spaces. ESAIM Control Optim. Calc. Var. 22(2), 355-370 (2016). DOI 10.1051/cocv/2015008. URL http://dx.doi.org/10.1051/cocv/2015008

10. Casas, E., Mateos, M., Rösch, A.: Finite element approximation of sparse parabolic control problems. Mathematical Control and Related Fields 7(3), 397-417 (2017). DOI 10.3934/ mcrf.2017014. URL http://dx.doi.org/10.3934/mcrf.2017014

11. Casas, E., Tröltzsch, F.: A general theorem on error estimates with application to a quasilinear elliptic optimal control problem. Comput. Optim. Appl. 53(1), 173-206 (2012). URL https://doi.org/10.1007/s10589-011-9453-8

12. Casas, E., Zuazua, E.: Spike controls for elliptic and parabolic PDEs. Systems Control Lett. 62(4), 311-318 (2013). DOI 10.1016/j.sysconle.2013.01.001. URL http://dx.doi. org $/ 10.1016 / \mathrm{j}$. sysconle.2013.01.001

13. Clason, C., Kunisch, K.: A duality-based approach to elliptic control problems in nonreflexive Banach spaces. ESAIM Control Optim. Calc. Var. 17(1), 243-266 (2011). DOI 10.1051/cocv/2010003. URL http://dx.doi.org/10.1051/cocv/2010003

14. Clason, C., Kunisch, K.: A measure space approach to optimal source placement. Comput. Optim. Appl. 53(1), 155-171 (2012). DOI 10.1007/s10589-011-9444-9. URL http://dx. doi.org/10.1007/s10589-011-9444-9

15. Herzog, R., Stadler, G., Wachsmuth, G.: Directional sparsity in optimal control of partial differential equations. SIAM J. Control Optim. 50(2), 943-963 (2012). DOI 10.1137/ 100815037. URL http://dx.doi.org/10.1137/100815037

16. Kunisch, K., Pieper, K., Vexler, B.: Measure valued directional sparsity for parabolic optimal control problems. SIAM J. Control Optim. 52(5), 3078-3108 (2014). DOI 10. 1137/140959055. URL http://dx.doi.org/10.1137/140959055

17. Lions, J.L.: Optimal control of systems governed by partial differential equations. Translated from the French by S. K. Mitter. Die Grundlehren der mathematischen Wissenschaften, Band 170. Springer-Verlag, New York-Berlin (1971)

18. Meidner, D., Vexler, B.: A priori error estimates for space-time finite element discretization of parabolic optimal control problems. I. Problems without control constraints. SIAM J. Control Optim. 47(3), 1150-1177 (2008). DOI 10.1137/070694016. URL http://dx.doi. org/10.1137/070694016

19. Meidner, D., Vexler, B.: A priori error estimates for space-time finite element discretization of parabolic optimal control problems. II. Problems with control constraints. SIAM J. Control Optim. 47(3), 1301-1329 (2008). URL https://doi.org/10.1137/070694028 
20. Meidner, D., Vexler, B.: Optimal error estimates for fully discrete galerkin approximations of semilinear parabolic equations. arxiv.org p. 1707.07889v1 (2017). URL https://arxiv . org/abs/1707.07889v1

21. Neitzel, I., Vexler, B.: A priori error estimates for space-time finite element discretization of semilinear parabolic optimal control problems. Numer. Math. 120(2), 345-386 (2012). DOI 10.1007/s00211-011-0409-9. URL http://dx.doi.org/10.1007/s00211-011-0409-9

22. Pieper, K.: Finite element discretization and efficient numerical solution of elliptic and parabolic sparse control problems. Ph.D. thesis, Technische Universität München (2015). URL http://mediatum.ub.tum.de/node?id=1241413

23. Pieper, K., Vexler, B.: A priori error analysis for discretization of sparse elliptic optimal control problems in measure space. SIAM J. Control Optim. 51(4), 2788-2808 (2013). DOI 10.1137/120889137. URL http://dx.doi.org/10.1137/120889137

24. Raviart, P.A., Thomas, J.M.: Introduction à l'analyse numérique des équations aux dérivées partielles. Collection Mathématiques Appliquées pour la Maîtrise. [Collection of Applied Mathematics for the Master's Degree]. Masson, Paris (1983)

25. de los Reyes, J.C., Meyer, C., Vexler, B.: Finite element error analysis for stateconstrained optimal control of the Stokes equations. Control Cybernet. 37(2), 251284 (2008). URL http://control.ibspan.waw.pl:3000/contents/export?filename= 2008-2-01_reyes_et_al.pdf

26. Stadler, G.: Elliptic optimal control problems with $L^{1}$-control cost and applications for the placement of control devices. Comput. Optim. Appl. 44(2), 159-181 (2009). DOI 10.1007/s10589-007-9150-9. URL http://dx.doi.org/10.1007/s10589-007-9150-9

27. Trautmann, P.: Measure valued optimal control problems governed by wave-like equations. Ph.D. thesis, University of Graz (2015)

28. Tröltzsch, F.: Optimal control of partial differential equations, Graduate Studies in Mathematics, vol. 112. American Mathematical Society, Providence, RI (2010). DOI 10.1090/gsm/112. URL http://dx.doi.org/10.1090/gsm/112. Theory, methods and applications, Translated from the 2005 German original by Jürgen Sprekels

29. Wachsmuth, G., Wachsmuth, D.: Convergence and regularization results for optimal control problems with sparsity functional. ESAIM Control Optim. Calc. Var. 17(3), 858-886 (2011). DOI 10.1051/cocv/2010027. URL http://dx.doi.org/10.1051/cocv/2010027 